\title{
A Review of Obinutuzumab (GA101), a Novel Type II Anti-CD20 Monoclonal Antibody, for the Treatment of Patients with B-Cell Malignancies
}

\author{
Kensei Tobinai $(\mathbb{D}) \cdot$ Christian Klein · Naoko Oya $\cdot$ Günter Fingerle-Rowson
}

Received: September 8, 2016/Published online: December 21, 2016

(c) The Author(s) 2016. This article is published with open access at Springerlink.com

\begin{abstract}
Obinutuzumab (GA101) is a novel, type II, glycoengineered, humanized anti-CD20 monoclonal antibody that has been developed to address the need for new therapeutics with improved efficacy in patients with lymphocytic leukemia and lymphoma of B-cell origin. Obinutuzumab has a distinct mode of action relative to type I anti-CD20 antibodies, such as rituximab, working primarily by inducing direct
\end{abstract}

Enhanced content To view enhanced content for this article go to http://www.medengine.com/Redeem/ 3527F060596B01CD.

K. Tobinai $(\bowtie)$

Department of Hematology, National Cancer Center

Hospital, Tokyo, Japan

e-mail: ktobinai@ncc.go.jp

C. Klein

Roche Pharmaceutical Research and Early

Development, Roche Innovation Center Zurich,

Schlieren, Switzerland

N. Oya

Oncology Lifecycle Management Department,

Chugai Pharmaceutical Co. Ltd, Tokyo, Japan

G. Fingerle-Rowson

Pharma Development Oncology, F. Hoffmann

La-Roche Ltd, Basel, Switzerland cell death and antibody-dependent cell-mediated cytotoxicity. Obinutuzumab is under investigation in a wide-ranging program of clinical trials in patients with B-cell malignancies. Efficacy as monotherapy has been reported in patients with relapsed/ refractory indolent and aggressive non-Hodgkin lymphoma (NHL) and in chronic lymphocytic leukemia (CLL) of B-cell origin. Improved outcomes have also been noted when obinutuzumab is added to chemotherapy in patients with B-cell NHL, and superiority over rituximab has been reported with combination therapy in patients with CLL. Ongoing research is focusing on developing options for chemotherapy-free treatment and on new combinations of obinutuzumab with novel targeted agents.

Keywords: Antibody-dependent cell-mediated cytotoxicity; B-cell lymphoma; CD20; Chronic lymphocytic leukemia; Glycoengineering; Monoclonal antibody; Non-Hodgkin lymphoma; Obinutuzumab; Oncology; Rituximab 


\section{INTRODUCTION}

Lymphocytic leukemia and lymphomas are malignant neoplasms that disrupt normal lymphocyte development and function. Lymphocytic leukemia originates in bone marrow and is characterized by high numbers of abnormal lymphocytes in the blood [1]. Chronic lymphocytic leukemia (CLL) is a slowly progressing disease that affects $\mathrm{B}$ lymphocytes (B-cells), mainly in older adults, and is the most common form of leukemia in the Western world, with an annual incidence rate of 4.2 per 100,000 [2]. Lymphomas, on the other hand, encompass a group of hematologic malignancies that arise mainly from mature $\mathrm{T}$ lymphocytes ( $\mathrm{T}$ cells) or B-cells in secondary lymphoid tissue, particularly the lymph nodes [3, 4]. They are subdivided based on the presence of Reed-Sternberg (or Hodgkin) cells into Hodgkin lymphoma (HL) and non-Hodgkin lymphoma (NHL) [5], of which NHL is by far the most common (accounting for around 90\% of cases) [6].

There are many types of NHL, differing in terms of microscopic appearance, growth patterns, clinical impact, and treatment $[3,4]$. However, NHL can be broadly divided into two major groups, B-cell and T-cell NHLs, of which the B-cell type accounts for approximately 85\% of all cases [5]. Indolent NHL (iNHL) is a slow-growing form of B-cell NHL that includes follicular lymphoma (FL) and marginal zone lymphoma, whereas more aggressive and faster-growing presentations of NHL include diffuse large B-cell lymphoma (DLBCL), Burkitt's lymphoma, and mantle cell lymphoma (MCL) [6].

FL is the most common type of iNHL, with an annual incidence that has risen from 2 to 3 per 100,000 in the 1950 s to 5 to 7 per 100,000 more recently in Western Europe [7]. Median age at diagnosis is in the 6 th decade of life, but up to $25 \%$ of patients with FL are aged 40 years or younger [8]. Approximately $55-70 \%$ of patients have bone marrow involvement at presentation, which is indicative of advanced disease [9-11]. Among aggressive subtypes of NHL, DLBCL is the most prevalent [12], having a crude annual incidence in Europe of 3.8 per 100,000 and accounting for between $30 \%$ and $58 \%$ of all cases of NHL [13]. Incidence of DLBCL increases with age, and risk factors include a family history of lymphoma, autoimmune disease, human immunodeficiency virus or hepatitis $\mathrm{C}$ virus infection, high body mass in young adulthood, and some types of occupational hazard exposure [13].

Management of NHL and CLL depends on stage, tumor burden, and other patient factors. For example, recent guidelines state that patients with early (stage I-II) iNHL should be offered radiotherapy [8, 14, 15], which can result in 10-year overall survival (OS) rates of up to $80 \%[14,15]$. However, most patients with iNHL are incurable [15], and successive relapses and increasing resistance to treatment characterize the individual course of the disease. Until the late 1990s (i.e., the period preceding the introduction of antibody therapy), these patients were usually treated with increasingly intensive combination chemotherapy regimens to reduce tumor burden and palliate symptoms. Response rates to conventional chemotherapy generally exceeded 50\% [12], and maximal tumor reduction could be achieved with high-dose chemotherapy and autologous stem cell support, but there were no apparent improvements in failure-free survival [16]. Treatment of symptomatic CLL and the more aggressive forms of NHL have also traditionally relied heavily on intensive combination chemotherapy. 
The therapeutic landscape for all forms of B-cell NHL changed markedly in the late 1990s with the approval of a CD20-directed monoclonal antibody (mAb), rituximab, after demonstration of significant activity as a single agent in patients with iNHL $[17,18]$. The antigen CD20 is a membrane protein found on the surface of all mature B-cells that typically has a constitutive and constant expression and therefore provides an excellent therapeutic target [19]. Moreover, it is found in 95\% of B-cell malignancies [20]. Rituximab acts by engaging Fc receptors on immune effector cells, such as natural killer (NK) cells and macrophages, and mediating complement-dependent cytotoxicity (CDC) and antibody-dependent cell-mediated cytotoxicity/phagocytosis (ADCC/ADCP), in addition to exerting direct antiproliferative and pro-apoptotic effects [12, 21].

The development of rituximab initiated the introduction of targeted immunotherapy for the treatment of indolent and aggressive forms of B-cell NHL and CLL and improved the general prognosis for patients with these diseases [9]. For instance, using a series of patients (with previously untreated stage I-II FL) from Stanford University, Tan et al. described four eras of FL treatment from 1960 to 2003: pre-anthracycline (1960-1975, 180 patients), anthracycline (1976-1986, 426 patients), aggressive chemotherapy/purine analogs (1987-1996; 471 patients), and rituximab (1997-2003, 257 patients) [22]. Median OS improved from 11 years in eras 1 and 2 to 18.4 years in era 3 ; at the time of reporting in 2013, median OS for era 4 had not been reached [22]. The development of rituximab in combination with chemotherapy represented a major step forward, significantly improving survival outcomes in not only patients with FL [23] but also in other
CD20-positive B-cell malignancies, including DLBCL [24, 25] and CLL [26]. These advances led to the adoption of rituximab as standard of care in patients with CLL, FL, and DLBCL who require systemic therapy $[2,8,13,14]$.

Despite the major therapeutic advances brought about by rituximab [15, 27], relapse and development of resistance to treatment are eventually seen in the majority of B-cell NHL patients $[12,15,28]$. The disease course is characterized thereafter by an ongoing decrease in the quality and duration of response with each subsequent course of therapy [15]. Mechanisms of resistance that have been suggested include increased $\mathrm{mAb}$ metabolism, reduced tumor penetration, reduced $\mathrm{mAb}$ binding (via Fc $\gamma$ RIII polymorphisms), resistance to $\mathrm{mAb}$ effector mechanisms, complement depletion, abnormal lipid raft composition of some malignant B-cells, downregulation of pro-apoptotic proteins, and impairments in immune effector cell recruitment or function $[29,30]$.

CD20 'shaving' is another potential resistance mechanism to type I anti-CD20 mAbs, whereby mAb-CD20 complexes are rapidly removed from the surface of B-cells via monocytes/macrophages through a mechanism known as trogocytosis [31-33]. This resistance mechanism can lead to fewer cell-surface CD20 antigens (with a consequent reduction in anti-CD20 mAb binding) and a decrease in Fc-mediated effector functions as well as a reduced $\mathrm{mAb}$ half-life. Additionally, there is evidence to indicate that Fc $\gamma$ RIIb-mediated internalization and degradation of the complex formed between the mAb and CD20 from the surface of some B-cell malignancies may cause resistance to type I anti-CD20 mAbs, such as rituximab [34-36], although this process of CD20 downregulation appears to be slower 
than trogocytosis [32]. This mechanism of mAb-CD20 internalization limits the engagement of natural effectors, reduces $\mathrm{mAb}$ half-life, and increases mAb turnover [37]. mAb-CD20 internalization correlates strongly with cis expression of the inhibitory Fc receptor Fc $\gamma$ RIIb on target B-cells and has been shown to predict less durable responses to rituximab therapy in patients with MCL [37].

Management of relapse and resistance in rituximab-treated patients presents a significant challenge [6], and there is a need for treatments with improved activity across B-cell NHL subtypes and CLL. Better understanding of antibody biology and modes of action, together with increased ability to design highly efficient therapeutics, has led to the development of novel mAbs with improved activity. As a review article, the following paper does not contain any new studies with human or animal subjects performed by any of the authors.

\section{OBINUTUZUMAB, A NOVEL HUMANIZED TYPE II MAB}

Obinutuzumab (GA101) is a novel, type II, glycoengineered, humanized anti-CD20 mAb that has been developed to address the need for novel therapeutics with higher activity than rituximab. The post-translational glycoengineering process used in the development of this agent [resulting in the absence of a fucose sugar residue from immunoglobulin G (IgG) oligosaccharides in the $\mathrm{Fc}$ region of the mAb molecule] was developed to increase activity by enhancing binding affinity to the Fc $\gamma$ RIII receptor on immune effector cells (Fig. 1) [38, 39]. Additionally, obinutuzumab has a modified

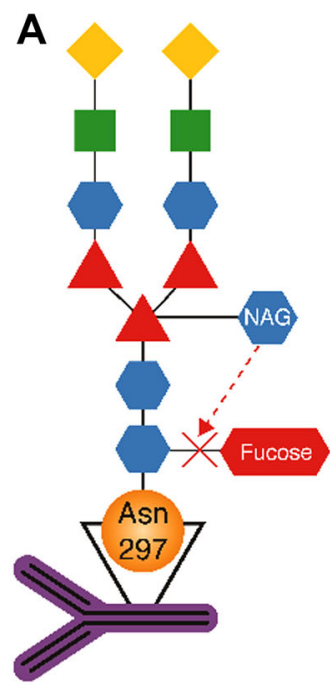

B

Fig. 1 Structure and binding behavior of obinutuzumab. Glycoengineered structure and type II binding properties of obinutuzumab. a Glycoengineering by defucosylation of immunoglobulin $\mathrm{G}$ oligosaccharides in the $\mathrm{Fc}$ region of obinutuzumab. In Chinese hamster ovary producer cells, $\mathrm{N}$-acetylglucosamine (NAG) is assembled into oligosaccharides, which sterically prevents the addition of fucose to the carbohydrate attached to asparagine (Asn)
297. b Hypothetical model of CD20 binding properties of type I and II antibodies. In contrast to inter-tetrameric CD20 binding of type I antibodies, intra-tetrameric binding of type II antibodies to CD20 does not lead to Fc $\gamma$ RIIb-mediated internalization of CD20 in lipid rafts (reproduced from Goede et al. [38] with permission; copyright (C) 2015 Karger Publishers, Basel, Switzerland) 
elbow-hinge amino acid sequence compared to type I agents, which together with the unique epitope recognized by obinutuzumab results in spatial alterations of the CD20-mAb complex on B-cells $[39,40]$; this is believed to be the molecular basis for the type II biology of obinutuzumab [40] as both type II character and cell death induction (as described below) can be switched on and off by mutating this elbow-hinge region [39].

The type II mechanism of action of obinutuzumab together with glycoengineering acts to enhance direct cell death and ADCC/ ADCP, while decreasing CDC (Fig. 2) [41], and differentiates the drug from classical type I anti-CD20 mAbs, such as rituximab and ofatumumab [39, 40, 42-44]. Rituximab, by comparison, works primarily via CDC (by clustering CD20 within lipid rafts) and by ADCC/ADCP, with direct cell death contributing much less to the overall antitumor activity [45]. Ofatumumab also acts primarily via CDC after binding both loop domains of $\mathrm{CD} 20$ at a different epitope compared to rituximab [46].

\section{Increased Direct Cell Death Induction}

Obinutuzumab has been shown to be faster than and superior to both rituximab and ofatumumab in inducing direct cell death in malignant B-cells. This was demonstrated by phosphatidylserine exposure and propidium iodide staining [with analysis by
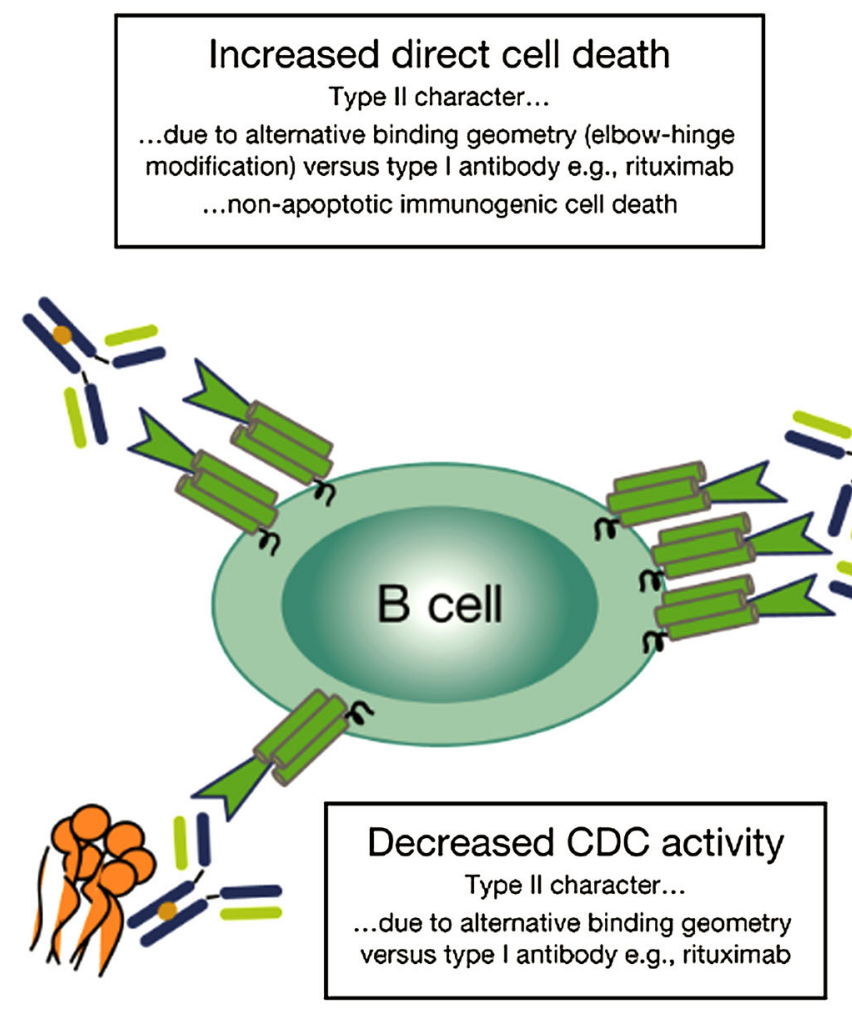

Fig. 2 Putative mechanisms of action of obinutuzumab. Please refer to the text for further information and supporting references. $A D C C$ antibody-dependent cell-

\section{Increased effector cell-mediated $A D C C$ and $A D C P$ \\ Increased affinity for FcyRIII receptors on immune effector cells... \\ ...due to glycoengineered Fc region}

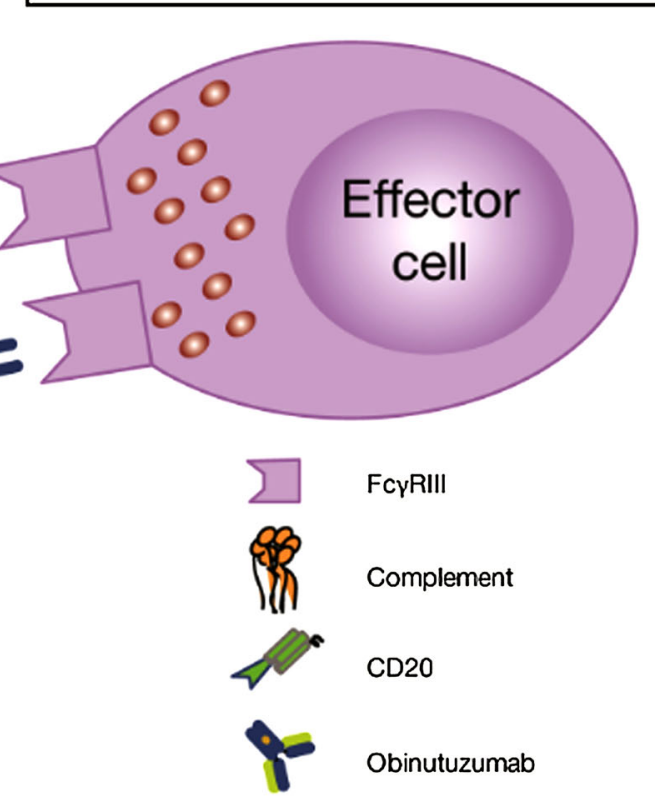

mediated cytotoxicity, $A D C P$ antibody-dependent cellular phagocytosis, $C D C$ complement-dependent cytotoxicity (adapted from Goede et al. [41] with permission) 
fluorescence-activated cell sorting (FACS) and time-lapse microscopy] in a panel of CD20-expressing tumor cell lines [39, 43]. While some researchers have questioned the validity of using FACS to assess mAb-induced direct cell death (because of potential mechanical interference with mAb-mediated homotypic adhesion) [47], multiple studies, using a variety of methods (including FACS), have confirmed that, overall, obinutuzumab induces greater direct cell death than type I mAbs [48-53] and occurs without disruption of homotypic aggregates [43, 54].

The mechanisms that may underlie the ability of type II anti-CD20 mAbs to directly evoke programmed cell death (PCD) are still poorly understood, but have been investigated in several studies [39, 48, 55]. Honeychurch et al. demonstrated actin-dependent, lysosome-mediated induction of PCD by type II mAbs, such as obinutuzumab or tositumomab, which was directly correlated with the production of reactive oxygen species (ROS) [48]. In contrast, type I mAbs, such as rituximab, induced only minimal levels of ROS and PCD. Generation of ROS mediated by nicotinamide adenine dinucleotide phosphate oxidase, independently of mitochondria, was unaffected by B-cell lymphoma 2 (BCL-2) overexpression and took place downstream of mAb-induced actin cytoskeletal reorganization and lysosome membrane permeabilization. The results thus indicated a newly characterized cell death pathway that is independent of the classical hallmarks of apoptosis and has the potential to bypass mechanisms of apoptotic resistance, thereby eliminating malignant cells that are refractory to conventional chemo- or immunotherapy. A similar mechanism of cell death has also been described for other antibodies targeting B-cell surface antigens, such as HLA-DR or CD37 [56, 57]. In a separate study, Cheadle and colleagues found that PCD induced by type II agents such as obinutuzumab is a form of immunogenic cell death that is characterized by the release of damage-associated pattern molecules, including heat shock protein 90 and adenosine triphosphate. It is believed that this mechanism of PCD could enhance the immune response by inducing dendritic cell mutation and subsequent $\mathrm{T}$-cell proliferation [49].

In contrast to these data, which are largely based on NHL cell lines, ex vivo studies utilizing CLL primary samples suggest that direct cell death induction is not the major mechanism of obinutuzumab-mediated B-cell depletion in CLL [50, 55, 58-61]. This observation may be a consequence of the lower proliferative state of non-stimulated CLL cells, as compared to NHL cell lines. Thus, in CLL, immune effector-mediated mechanisms (as described below) may play a more important role than direct cell death induction in mediating the antiproliferative effects of obinutuzumab. In line with this assumption, a study has demonstrated that CD40 stimulation can sensitize CLL cells to lysosomal cell death induction by obinutuzumab, providing evidence that drug sensitivity in CLL cells can be modulated by microenvironmental stimuli [55].

\section{Complement-Dependent Cytotoxicity}

Antibody-mediated CDC is initiated by fixing of C1q (the initiating component of the classical complement pathway) to the Fc portion of target-bound antibodies. This triggers a cascade that in turn leads to the formation of C3 and C5 convertase and ultimately to the membrane attack complex (MAC). The MAC then causes cell lysis by disrupting the plasma membrane of the target cell [62]. 
The ability of CD20 antibodies to mediate CDC appears to be determined by the effect of $\mathrm{mAb}$ binding and cross-linking on the redistribution of the target antigen into lipid rafts on the surface of the target cell. The density and positioning of the antigen-antibody complexes influence $\mathrm{C} 1 \mathrm{q}$ binding and, in turn, the complement cascade [62]. Complement recruitment as such is a key characteristic distinction between type I CD20 mAbs, such as rituximab and ofatumumab, which mediate strong CDC, and type II CDC antibodies like obinutuzumab, which only mediate weak CDC activity in cellular assays $[39,42,43]$. CDC is therefore not thought to contribute meaningfully to the overall activity of obinutuzumab [38, 62]. Interestingly, the limited capacity of obinutuzumab to fix complement via its Fc portion may further enhance its capability to bind to Fc $\gamma$ RIII and mediate ADCC [63].

\section{ADCC/ADCP}

Glycoengineering as well as subsequent enhancement of affinity for Fc $\gamma$ RIIIa (an activating Fc receptor expressed primarily on NK cells) was confirmed as the predominant determinant of the superior ADCC activity of obinutuzumab over type I anti-CD20 mAbs in a series of experiments using NHL cell lines and human PBMCs expressing the V158/V158 or F158/F158 Fc $\gamma$ RIIIa receptor [43]. While obinutuzumab was superior to rituximab and ofatumumab in terms of potency and overall cell killing, the ADCC activity of a non-glycoengineered version of obinutuzumab was similar to that of rituximab and ofatumumab, confirming the additional benefit conferred by glycoengineering [43]. Notably, the induction of ADCC was found to be particularly more potent with obinutuzumab than rituximab in the presence of nonspecific human IgG at physiological concentrations, as found in human blood [39].

In an assessment of ADCP, Herter et al. used FACS analysis to show that obinutuzumab, rituximab, and ofatumumab have comparable overall phagocytic activity in NHL cell lines and primary human monocyte-derived macrophages (MDMs) [44]. Rafiq et al. have demonstrated the phagocytic activity of obinutuzumab against membrane-dyed CLL cells undergoing flow cytometry, although ofatumumab and rituximab produced greater phagocytosis than obinutuzumab in these experiments [50]. In a recent report, intravital imaging revealed improved Kupffer cell-mediated phagocytosis of B-cells as an important in vivo mode of action of glycoengineered anti-CD20 mAbs, such as obinutuzumab, which underlies their improved activity compared with non-Fc-engineered antibodies [64].

ADCC is carried out mainly by NK cells, which carry inhibitory killer cell immunoglobulin-like receptors (KIRs) that interact with exposed epitopes on the class I human leukocyte antigen (HLA). Experiments using peripheral blood mononuclear cells (PBMCs) from healthy donors, primary CLL cells from patients, and a variety of cell lines have shown that, because of its Fc modification, obinutuzumab can compensate for inhibitory KIR/HLA interactions [65]. This results in the recruitment of additional NK cells for ADCC, and target cell depletion is not negatively impacted by KIR/HLA interactions [65]. Genotyping of patients participating in the CLL11 study (comparing chlorambucil, rituximab-chlorambucil, and obinutuzumab-chlorambucil in previously untreated patients with CLL) has shown that the prognosis for patients with a low number of KIR/HLA interactions is better than that for 
Fig. 3 Percentage tumor growth inhibition (TGI) in combination studies of mouse Z138 MCL xenografts [69]. *Statistically significant $(p<0.001$; Tukey-Kramer test) vs. single-agent treatments.**Statistically significant vs. G monotherapy and RIT + FLU. ${ }^{* * *}$ Statistically significant vs. RIT monotherapy. TGI was calculated from tumor volume (TV) $\quad\left[\left(\right.\right.$ length $\times$ width $\left.\left.^{2}\right) / 2\right]$, calculated from staging until study termination, as follows: TGI $(\%)=100-\frac{\text { Median }\left[\mathrm{TV}(\text { treated })_{\text {day } z}-\mathrm{TV}(\text { treated })_{\text {day } x}\right]}{\text { Median }\left[\mathrm{TV}(\text { respective control })_{\text {day } z}-\mathrm{TV}(\text { respective control })_{\text {dayx } x}\right]} \times 100$.

Each treatment group was compared with its respective vehicle control. TVday $z$ represented TV for an individual animal at a defined study day (day $z$ ), and TVday $x$ represented TV of an individual animal at the staging day (day $x$ ). Animals in control groups received $0.9 \%$ sodium chloride vehicle; randomization to treatments took place 9-27 days after tumor cell injection. Animals were killed at various time points from day 30 to day 66. BEN bendamustine, $C H L$ chlorambucil, FLU fludarabine, $G$ obinutuzumab (GA101), MCL mantle cell lymphoma, RIT rituximab, TGI tumor growth inhibition

patients with a higher number of such interactions [66]. Taken together, these results indicate that ADCC is a key mode of action for obinutuzumab and is influenced by the number of the inhibitory interactions.

Unlike type I anti-CD20 mAbs, type II agents are found to undergo reduced CD20 internalization (Fig. 1) [67]. Indeed, increased stability of surface-accessible CD20 was noted with obinutuzumab relative to both rituximab and ofatumumab using FACS in a human DLBCL cell line and in blood from patients with CLL [43]. Ultimately, this may further enhance the immune effector cell-mediated mechanisms of type II anti-CD20 mAbs, independently of, but in conjunction with, glycoengineering. Furthermore, type II anti-CD20 mAbs may be less susceptible to the development of resistance mechanisms affecting effector-cell-mediated cytotoxicity. The impact of trogocytosis on reducing the efficacy of type II mAbs through shaving of CD20 molecules from the cell surface requires further investigation.

\section{Superior and/or Faster Whole Blood B-cell Depletion}

Measurement of overall B-cell depletion in whole blood allows all mechanisms of action of therapeutic antibodies to be assessed in a single assay. Potent B-cell depleting activity in peripheral blood and lymphoid tissue superior to that of rituximab has been reported with obinutuzumab in non-human primate models [39]. In addition, Mössner et al. showed obinutuzumab to be significantly (10-25 times) more potent and 1.5-2.5 times more effective $(p<0.001)$ than rituximab in depleting B-cells in whole blood from healthy human donors [39]. These results were then confirmed using primary malignant B-cells from a patient with CLL [39]. Superior B-CLL cell depletion in whole blood with obinutuzumab was demonstrated independently by several other research groups [50, 58, 60, 61], whereas Bologna et al. showed that obinutuzumab treatment depleted B-cells in whole blood from CLL patients to a similar extent to rituximab, but at a much faster rate [59]. These findings may reflect the different experimental methods that were used but overall demonstrate the enhanced B-cell-depleting activity of obinutuzumab. Recently, Ysebaert et al. described the effects of rituximab and obinutuzumab on B-cell depletion in 96 CLL patient samples with different prognostic factors [68]. Median proportions of B-cell depletion after whole-blood assay were $22 \%$ with rituximab and $63 \%$ with obinutuzumab $(p<0.001)$, independent of their prognostic factors [68]. 

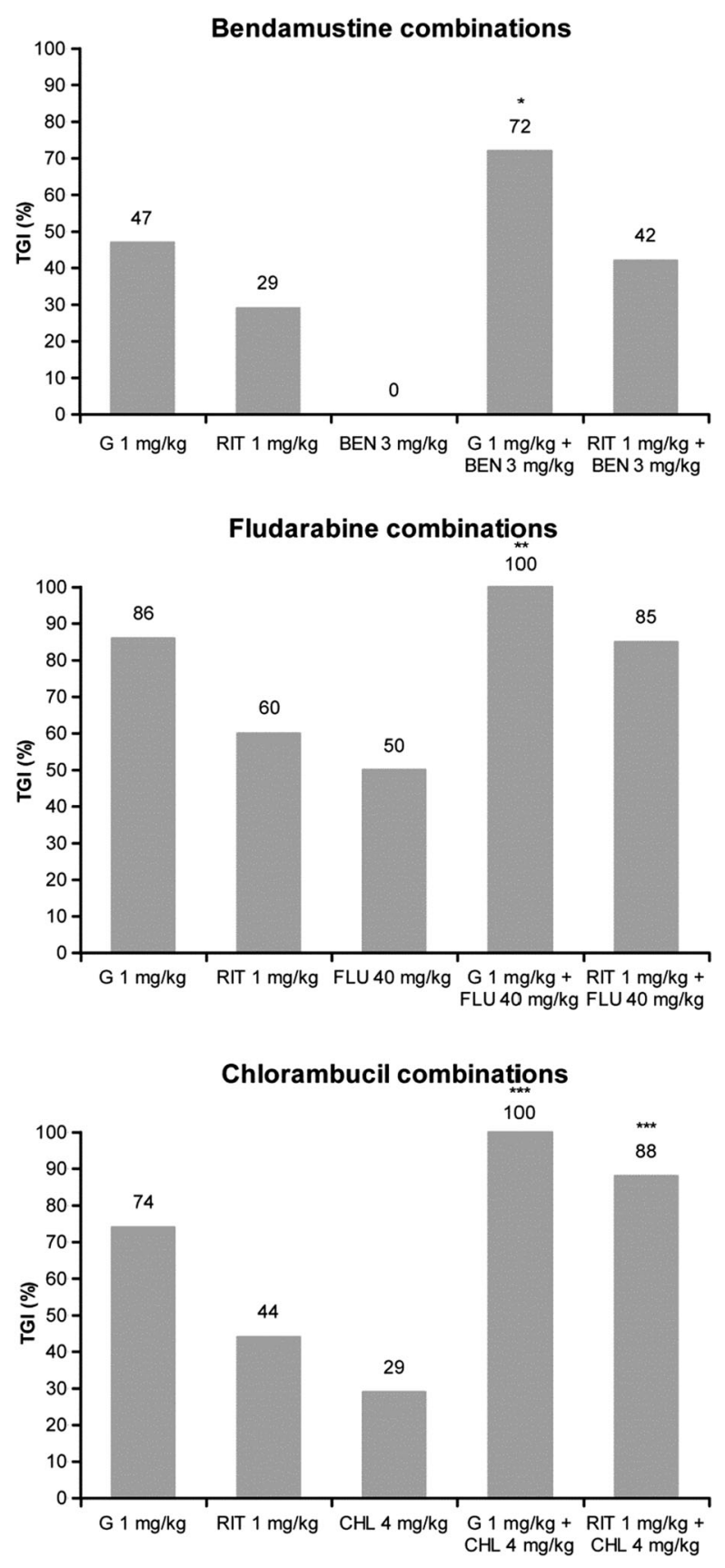

\section{Superior Antitumor Efficacy In Vivo}

Finally, obinutuzumab has shown superiority over rituximab in vivo in various human lymphoma xenograft models [39]. Dose-dependent efficacy in the range of $1-30 \mathrm{mg} / \mathrm{kg}$ was noted in a staged aggressive SU-DHL4 DLBCL model, with complete tumor regression in all animals and lasting tumor eradication in $90 \%$ at the highest dose of $30 \mathrm{mg} /$ $\mathrm{kg}$. In contrast, tumor regression was not shown at any equivalent dose of rituximab [39] or with ofatumumab [43]. In this model, second-line obinutuzumab treatment was also effective in inhibiting the progression of tumors that had progressed under first-line treatment with rituximab and that were no longer responsive to rituximab [39] or ofatumumab [43]. Superior efficacy of obinutuzumab over rituximab or ofatumumab has also been shown in an aggressive disseminated MCL model $[39,43]$.

Herting et al. described the antitumor activity of obinutuzumab and rituximab alone and in combination with bendamustine, fludarabine, chlorambucil, doxorubicin, and cyclophosphamide/vincristine in subcutaneous murine xenograft models using Z138 MCL and WSU-DLCL2 DLBCL tumors [69]. As obinutuzumab had high single-agent activity in these models, suboptimal doses were used in order to observe combination effects. Superior tumor growth inhibition was achieved with obinutuzumab plus bendamustine over rituximab plus bendamustine, and statistically significant effects versus the respective single treatments were also observed (Fig. 3). In addition, obinutuzumab showed significantly greater activity than rituximab when combined with fludarabine, chlorambucil, or cyclophosphamide/ vincristine. Obinutuzumab monotherapy was as effective as, or more effective than, rituximab plus chemotherapy in vivo [69].

\section{CLINICAL TRIALS OF OBINUTUZUMAB IN B-CELL $\mathrm{NHL}$}

Obinutuzumab is now under evaluation in an extensive clinical trial program in patients with B-cell malignancies. 


\section{Obinutuzumab Monotherapy}

Obinutuzumab given as monotherapy has been investigated in the GAUGUIN study (NCT00517530), a multicenter phase Ib/II clinical trial in patients with B-cell malignancies [70-73]. The primary objective of the phase Ib part of the study was to investigate the safety and tolerability of escalating intravenous doses of obinutuzumab in patients with CD20-positive lymphoid malignancies, including NHL and CLL. This was followed by a phase II part to study efficacy and safety. Patients were adults aged 18 years or older with an Eastern Cooperative Oncology Group (ECOG) performance status of $0-2$.

The first two reports from GAUGUIN (Table 1) communicated phase I [70] and II [71] results from patients with relapsed/ refractory iNHL, most of whom had FL. In the phase I part, 21 patients (median of five prior therapies, 20/21 with prior rituximab) received escalating doses of obinutuzumab monotherapy over eight 21-day cycles. The majority of adverse events (AEs) were infusion-related reactions (IRRs), nearly all of which were of grade $1-2$ in severity (Table 1 ).

IRRs with obinutuzumab, like those seen with rituximab, have been attributed to cytokine release. In previous studies of rituximab, patients with IRRs were found to release greater amounts of interleukin (IL)-8, IL-6, and tumor necrosis factor-alpha than those without IRRs [76-78]. Patients with a baseline absolute lymphocyte count of $\geq 50 \times 10^{9} / 1$ appear to have a particularly high risk of developing IRRs [76-78]. The incidence and severity of IRRs in patients treated with obinutuzumab is reportedly greater than with rituximab and has been linked to immediate and marked release of IL- 6 and IL- 8 that is limited to the first infusion and is accompanied by rapid destruction of circulating B-cells and disappearance of circulating NK cells from the peripheral blood [79].

In GAUGUIN, 18 grade 3-4 AEs occurred in 7 patients; all treatment-related grade 4 events were neutropenias, while most grade 3 AEs were hematologic events and IRRs. Notably, there were no dose-limiting toxicities (DLTs). Two of nine rituximab-refractory patients showed a tumor response, and the best overall response rate (ORR) was 43\%, with five complete responses (CRs) and four partial responses indicating the promising activity of obinutuzumab.

Phase II data from relapsed/refractory iNHL patients ( $n=40$; Table 1$)$ showed promising efficacy and acceptable tolerability, particularly in the higher dose group (1600/800 mg) over a median observation time of 33.7 months [71]. Of the phase II patients, $90 \%$ had stage III or IV disease and had received a median of two prior rituximab treatments (range 0-5); 45\% and 55\% were refractory to their last treatment or to rituximab, respectively. End-of-treatment ORRs in rituximab-refractory patients were $8 \%$ in the $400 / 400 \mathrm{mg}$ group and 50\% in the $1600 / 800 \mathrm{mg}$ group. The two CRs were seen in the higher dosage group.

The phase II GAUGUIN study for patients with aggressive forms of B-cell NHL showed similar tolerability and promising efficacy (Table 1) [72]. These patients (25 with DLBCL and 15 with MCL) had received a median of three prior treatments (range 1-17), and 25 (63\%) were rituximab-refractory. Of the 25 rituximab-refractory patients, 4 (16\%) had objective responses to induction treatment and one responded during follow-up. Four of the five responses were achieved at the $1600 / 800 \mathrm{mg}$ dose. The best ORR in all patients with aggressive B-cell NHL was 30\%. Median response duration (all responders) was 


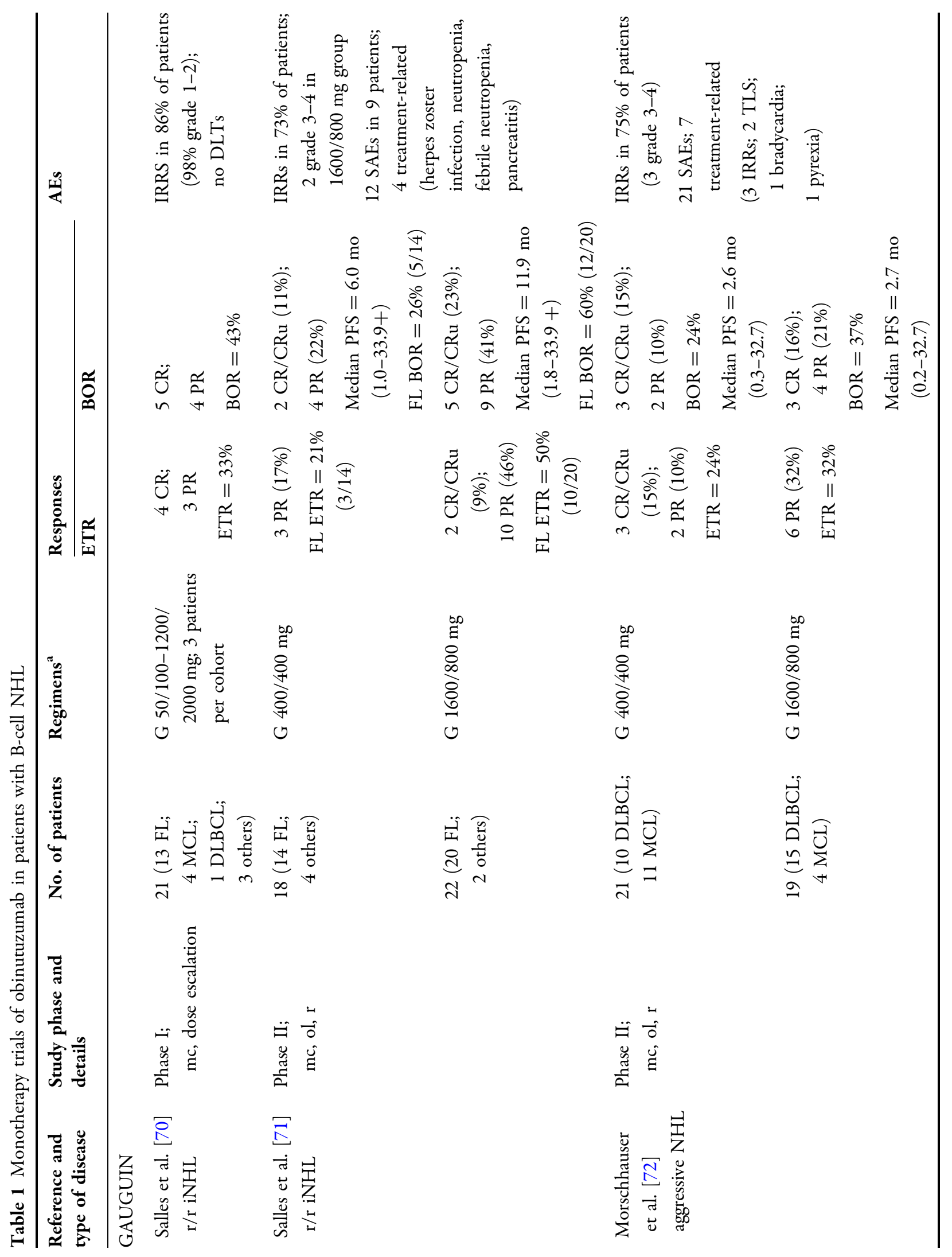




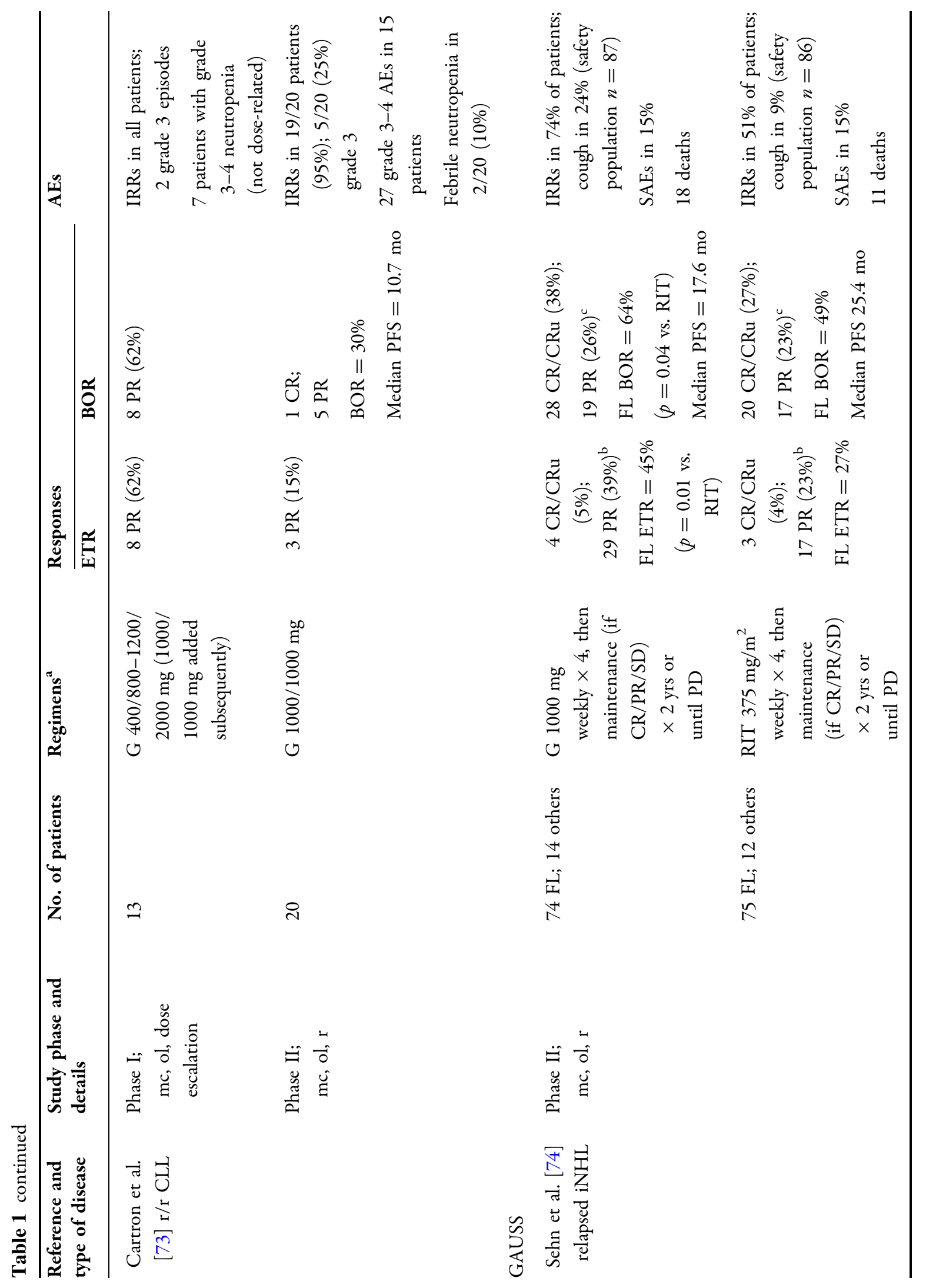




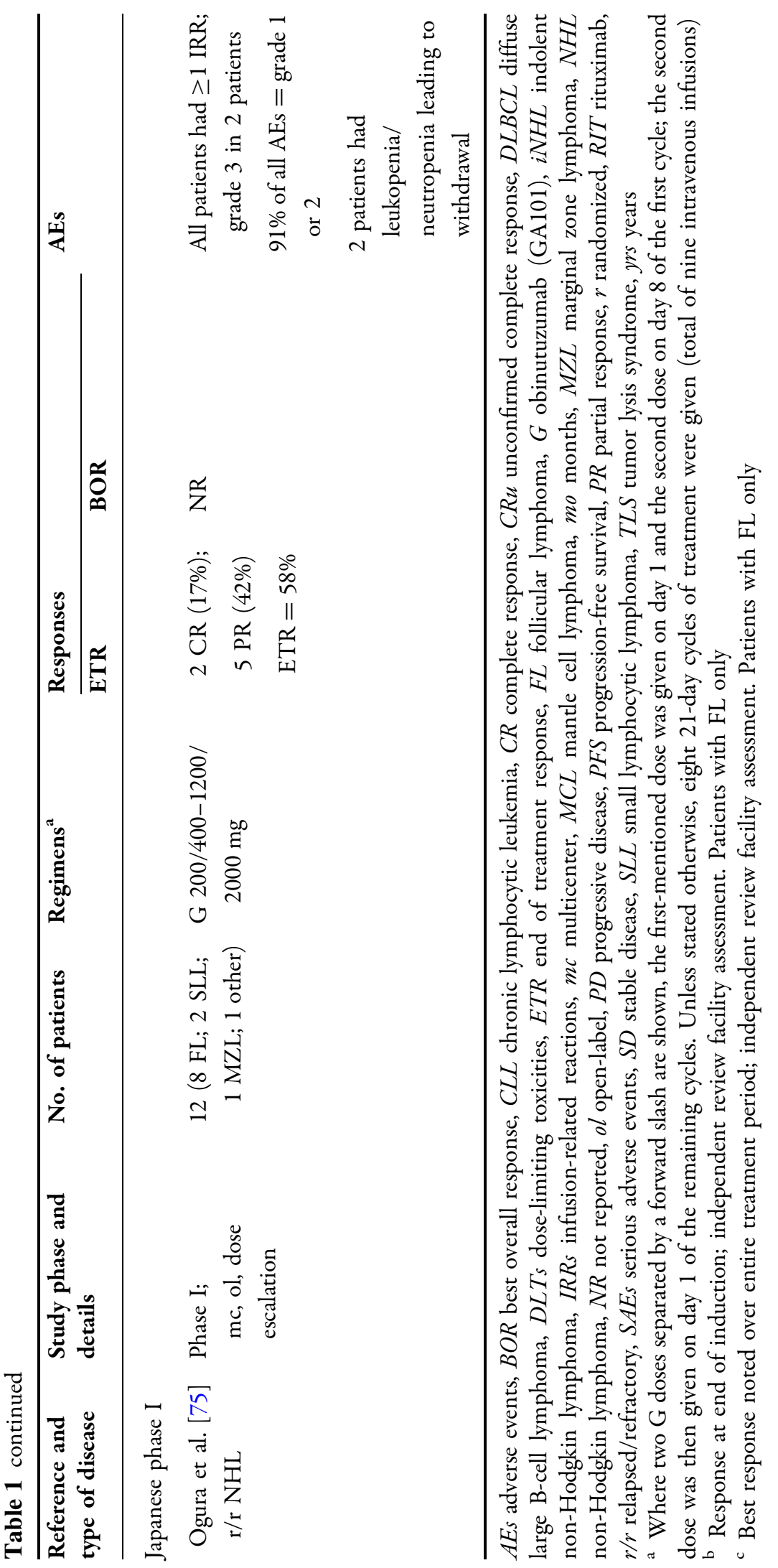


9.8 months; there was no relevant difference in progression-free survival (PFS) between dosage groups after a median of 14.2 months (range 0.3-36.1 months) of observation. Based on both these results and on pharmacokinetic modeling, which showed that obinutuzumab $1000 \mathrm{mg}$ per cycle with additional $1000 \mathrm{mg}$ doses on days 8 and 15 of cycle 1 can achieve exposures similar to the $1600 / 800 \mathrm{mg}$ regimen used in GAUGUIN [80], a simplified flat-dose $1000 \mathrm{mg}$ schedule was adopted for subsequent phase II and III investigation [81]. It has been hypothesized that differences in CD20 binding, activation of biological pathways, and underlying mechanisms of action compared with type I anti-CD20 mAbs permit the use of flat dosing for obinutuzumab rather than conventional body surface area-based dosing [80]. The 1000-mg flat-dose schedule for obinutuzumab, which has been implemented as the standard dose in clinical trials, rapidly achieves CD20 target saturation in all patients tested, with serum concentrations maintained at this therapeutic level throughout the treatment course [80].

Among 13 phase I patients with relapsed/ refractory CLL in the separately reported GAUGUIN CLL study, there was a median response duration of 10.5 months (range 8.5-37 months) in eight partial responders (for a best ORR of $62 \%$; Table 1) [73]. Among 20 patients with CLL who were recruited to phase II, the best ORR was substantially lower $(6 / 20 ; 30 \%)$. This has been linked to a higher baseline tumor burden and consequent lower treatment exposure than in phase I [73]. Median response duration was 8.9 months (range 0.8-26.1 months). Tolerability was acceptable in other groups of patients, with IRRs being the most common AEs (Table 1). Most notably, all CLL patients treated in the GAUGUIN study experienced a rapid and sustained elimination of
B-cells in the peripheral blood, which was independent of the dose applied. These findings provided the rationale for investigating obinutuzumab in the phase III CLL11 trial. Data from CLL11 and other dedicated studies in patients with CLL are presented later in this review.

Further data in larger numbers of patients with relapsed iNHL were obtained from the multicenter GAUSS study (NCT00576758) [74]. The initial phase I component of this trial evaluated obinutuzumab doses of 200-2000 mg given once weekly for 4 weeks (induction) followed by maintenance therapy every 3 months for 2 years in 22 patients with relapsed B-cell NHL (including 10 with FL) or CLL [82]. The best ORR was 32\%, with a response observed in $15 \%$ of rituximab-refractory patients. The maximum tolerated dose was not reached. A flat dose of $1000 \mathrm{mg}$ was selected for the phase II part of GAUSS based on the phase I data and other clinical experience. As summarized in Table 1, 175 patients with relapsed iNHL were enrolled to phase II, of whom 149 had FL. The ORR at the end of induction was higher with obinutuzumab than with rituximab, as shown by investigator assessments at the end of the induction period (45\% vs. $33 \% ; p=0.08)$ and by a blinded independent review panel $(45 \%$ vs. $27 \%$; $p=0.01$ ) (Table 1$)$. There was no significant difference between treatments for the secondary endpoint of PFS, but the study was not powered to detect differences. AEs were balanced between the two groups, but there were more IRRs and coughs in the obinutuzumab group (Table 1) [74].

An additional phase I study in 12 Japanese patients, of whom 8 had FL, also showed no DLTs [75]. B-cell depletion was seen in all patients and persisted for the duration of treatment. No disease progression was observed during the treatment period. 
Moreover, the majority of AEs were of grade 1 or 2 in severity; as in other studies, IRRs predominated (Table 1).

\section{COMBINATION THERAPY}

\section{Phase I and II}

Following the promising activity of obinutuzumab in phase I and II single-agent studies, a number of studies have been carried out in the combination therapy setting. The phase Ib GAUDI study (NCT00825149) examined the safety and antitumor activity of two doses of obinutuzumab (G) (400/400 mg or 1600/800 mg) combined with cyclophosphamide, doxorubicin, vincristine, and prednisone (CHOP; G-CHOP), or with fludarabine and cyclophosphamide (FC; G-FC), as induction therapy in 56 patients with relapsed/refractory FL (Table 2) [83]. These patients had received up to six prior treatments. Patients were allocated to either CHOP or FC by their physicians on the basis of clinical need and the physician's judgment; allocation to the two obinutuzumab dose regimens was randomized. As seen in the previously described phase I and II monotherapy studies, IRRs were the most common AEs and were predominantly of mild severity (Table 2). Two patients from the G-FC $400 / 400 \mathrm{mg}$ arm and three from the G-FC $1600 / 800 \mathrm{mg}$ arm discontinued treatment because of AEs; one other patient in the latter group discontinued because of insufficient response. All rituximab-refractory patients responded to treatment, and the high ORRs noted (Table 2) supported future phase III investigation [83].

As G-CHOP was seen to have a safety profile similar to R-CHOP (rituximab combined with CHOP), the design of the GAUDI study was amended to compare the safety of obinutuzumab plus CHOP or bendamustine in
81 treatment-naïve patients with FL (Table 2) [84]. After 2 years' maintenance in initial responders who went on to receive obinutuzumab $1000 \mathrm{mg}$ as monotherapy, high $\mathrm{CR}$ rates were seen in both treatment arms and opportunistic infections were infrequent [84].

G-CHOP was also investigated in the first-line setting in 80 patients with advanced DLBCL in the GATHER study (NCT01414855) (Table 2) [85]. The ORR and CR rates as determined by investigators were $83 \%$ and $55 \%$, respectively (Table 2 ). IRRs, most of which were grade 1-2 in intensity, were typically observed during the first cycle.

\section{Phase III}

The phase III GADOLIN study (NCT01059630) (Table 2) compared bendamustine monotherapy $\left(120 \mathrm{mg} / \mathrm{m}^{2}\right.$ on day 1 and 2 , for up to six 28-day cycles) with obinutuzumab (1000 mg on day 1 , 8 , and 15 of cycle 1 and on day 1 of cycles $2-6$ in 28 -day cycles) plus bendamustine $\left(90 \mathrm{mg} / \mathrm{m}^{2}\right.$ on day 1 and 2, for up to six 28-day cycles) induction and obinutuzumab maintenance (1000 mg every 2 months for 2 years or until progression in patients achieving stable disease or better with induction) in 396 patients with rituximab-refractory iNHL who had received a median of two prior treatments [86]. At a preplanned interim analysis for efficacy, enrollment was stopped and the trial was analyzed in full, as the primary endpoint had been met. Median observation time was 20.3 months for bendamustine alone and 21.9 months for obinutuzumab plus bendamustine. Recruitment had been ongoing between data cutoff and interim analysis, and a significant number of patients were still undergoing treatment. PFS was significantly longer with combination therapy (median not reached) than with bendamustine alone 


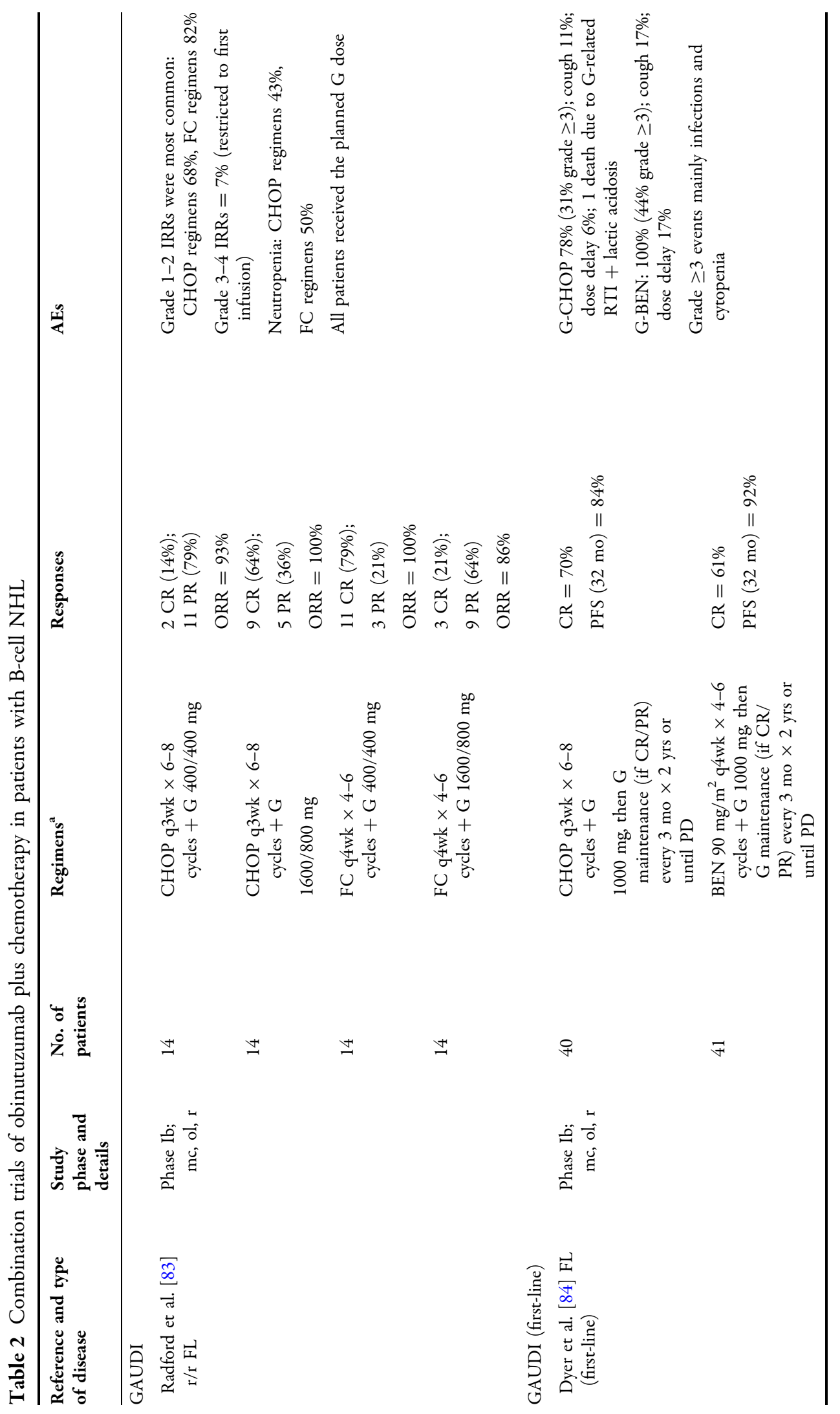




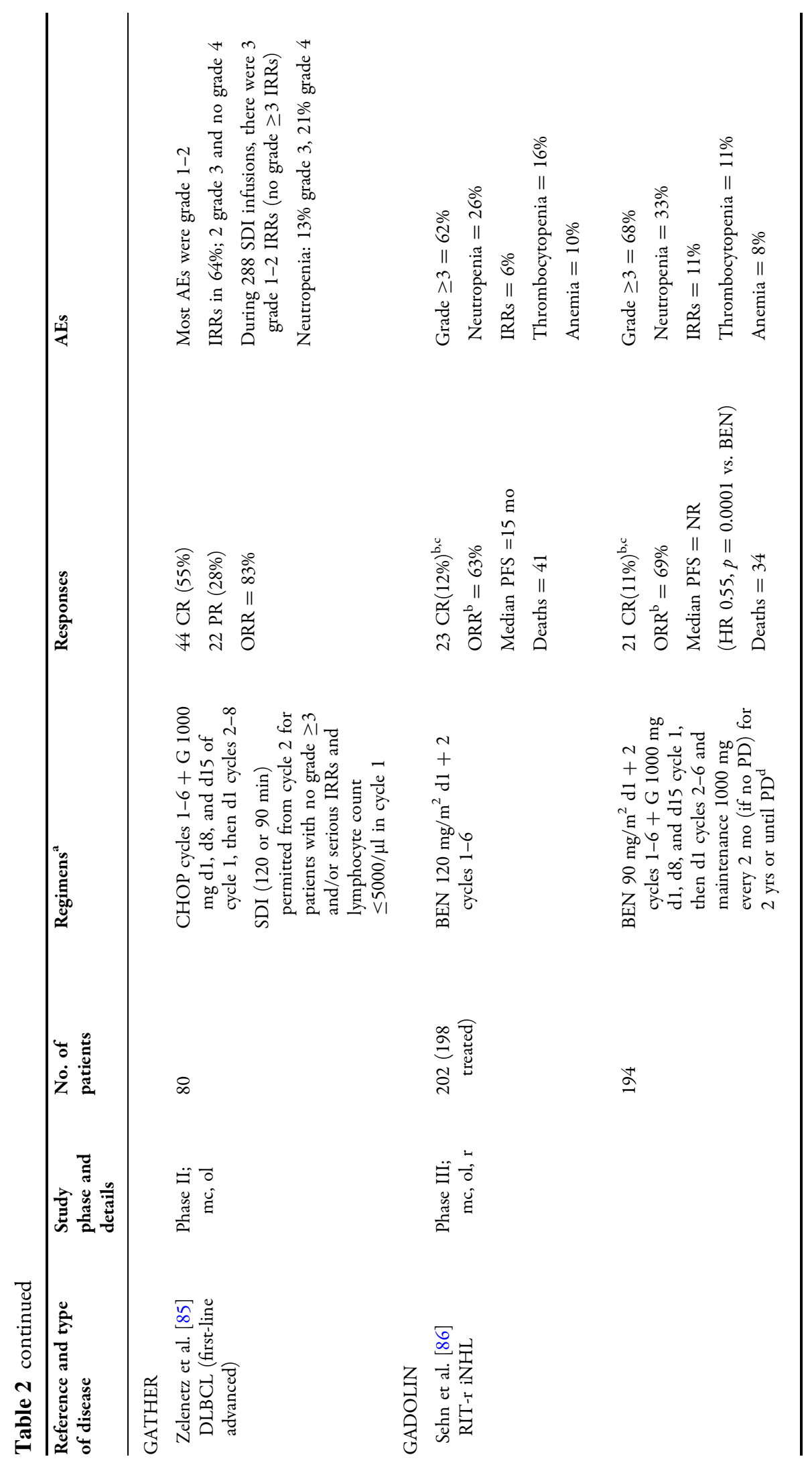




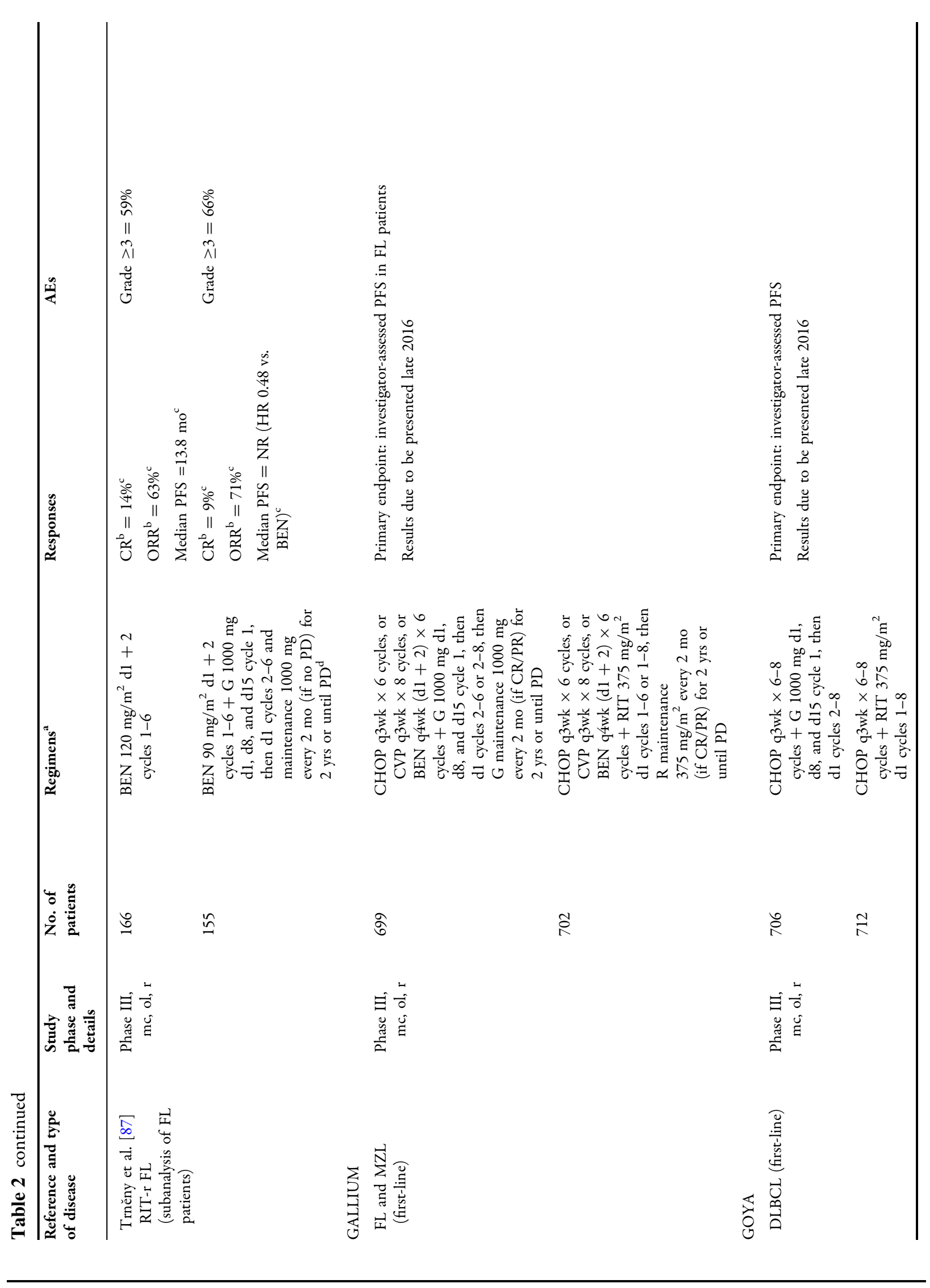




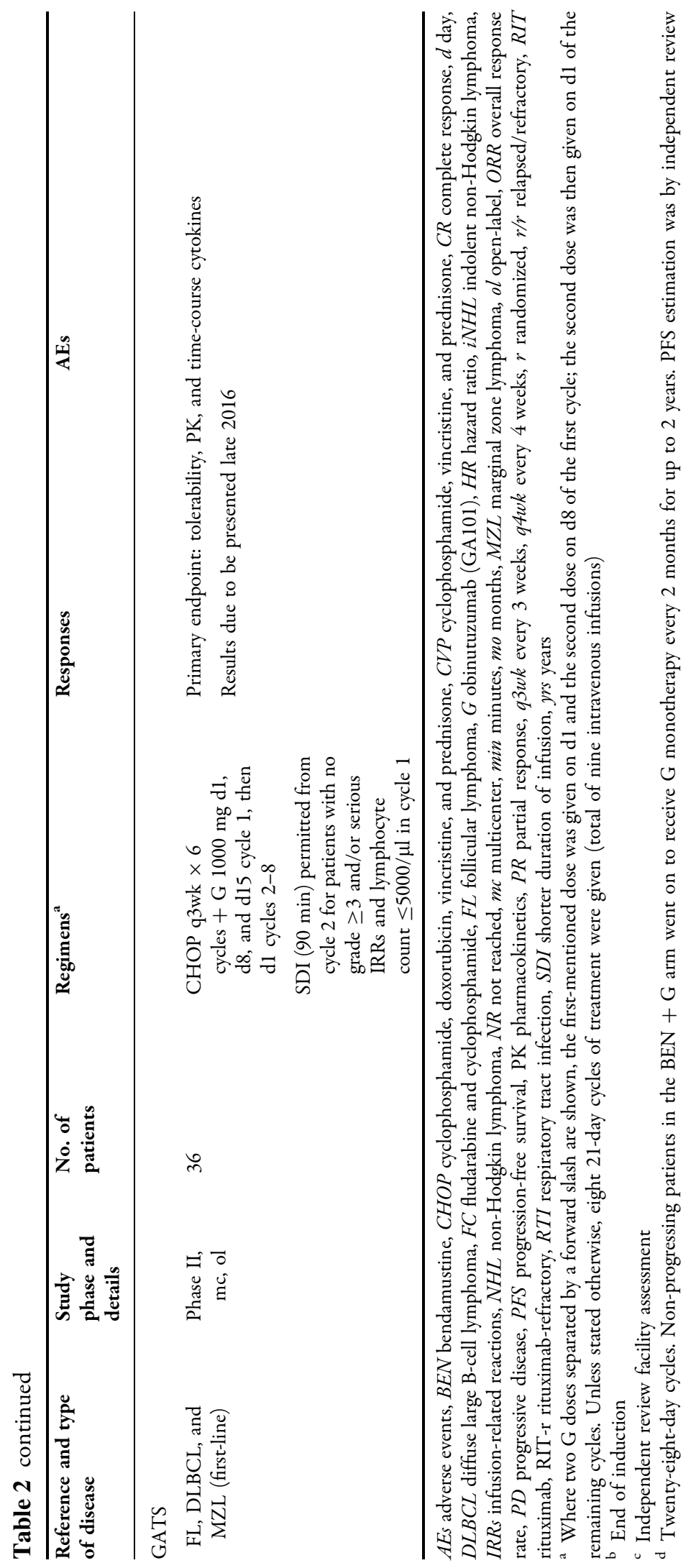


(median 14.9 months) as assessed by an IRF [primary endpoint; hazard ratio (HR) 0.55; 95\% confidence interval (CI) 0.40-0.74; $p=0.0001]$.

Subgroup analyses and secondary endpoints (including PFS by investigator and OS) were consistent and supportive of the primary endpoint. There were no significant differences in IRF-assessed ORR or CR rate at the end of induction. Whether this was due to the differences between groups in bendamustine dosage or to the different modes of action of obinutuzumab and bendamustine was uncertain (Table 2). The prognostic relevance of minimal residual disease (MRD) status in 93 biomarker-evaluable patients with $\mathrm{FL}$ in GADOLIN was also investigated [88]. MRD analysis measures the small number of malignant cells that remain after treatment. MRD status was associated with clinical CR rate and improved PFS, and significantly more patients were MRD-negative after induction with obinutuzumab plus bendamustine than after bendamustine alone $(82 \%$ vs. $43 \%$; $p<0.0001)$. Median OS was not reached in either arm at the time of reporting, but there were more deaths due to disease progression with bendamustine alone than with obinutuzumab plus bendamustine [86]. Overall, grade $\geq 3$ AEs were more common in the obinutuzumab plus bendamustine group, but grade $\geq 3$ thrombocytopenia and anemia were more frequent in the bendamustine-only group (Table 2).

Emerging data from GADOLIN also suggest improvements in health-related quality of life [89]. The time taken to $\mathrm{a} \geq 6$-point worsening from baseline on the Functional Assessment of Cancer Treatment-Lymphoma Trial Outcome Index (FACT-Lym TOI) was 8.0 months in the combination therapy arm and 4.6 months with bendamustine alone. A higher proportion of patients reported meaningful improvements on the lymphoma subscale, lymphoma TOI, and total score with obinutuzumab plus bendamustine than with bendamustine alone [89].

Ongoing phase III combination therapy studies with obinutuzumab include GALLIUM (first-line advanced iNHL; NCT01332968) and GOYA (first-line DLBCL; NCT01287741). GALLIUM aims to assess the efficacy and safety of obinutuzumab plus chemotherapy versus rituximab plus chemotherapy followed by maintenance immunotherapy. After induction, responders will progress to maintenance therapy with their randomized antibody treatment alone, given every 2 months until disease progression or for a maximum of 2 years. In May 2016, at a prespecified interim analysis, the GALLIUM Independent Data Monitoring Committee recommended analysis of the study data as the primary endpoint of investigator-reported PFS had been met [90]. This is the second head-to-head comparative trial against rituximab that has shown a positive result for obinutuzumab, the first being the CLL11 study (described below). In the phase III GOYA trial, previously untreated patients with DLBCL were randomized to obinutuzumab $1000 \mathrm{mg}$ every 21 days (with two additional doses on day 8 and day 15 of cycle 1) or rituximab $375 \mathrm{mg} / \mathrm{m}^{2}$ every 21 days for eight cycles in addition to $6-8$ cycles of CHOP chemotherapy. Recruitment of 1418 patients was completed in 2014. After the final analysis in July 2016, Roche issued a press release to say that the study's primary endpoint of improvement in investigator-assessed PFS had not been met. Detailed results are expected to be announced at ASH 2016. 


\section{CLINICAL TRIALS OF OBINUTUZUMAB IN CLL}

\section{Phase I and II}

A number of studies have investigated the use of obinutuzumab in patients with CLL. The GAGE study (NCT01414205), a randomized, phase II study in 80 symptomatic, untreated CLL patients, demonstrated significant antitumor activity with both $1000 \mathrm{mg}$ and $2000 \mathrm{mg}$ dosages of obinutuzumab. End of induction response was superior with the higher dosage (Table 3) [91]. The results suggested a possible dose-response relationship, but this requires further investigation and longer follow-up. Obinutuzumab has also shown manageable toxicity and promising activity in combination with either bendamustine or FC in the phase Ib GALTON study (NCT01300247) in 41 patients with previously untreated CLL [92].

\section{Phase III}

In the phase III CLL11 study (Table 3), 781 patients with previously untreated CLL and a score higher than 6 on the Cumulative Illness Rating Scale or an estimated creatinine clearance of $30-69 \mathrm{ml} / \mathrm{min}$ were randomized to chlorambucil alone, obinutuzumab plus chlorambucil, or rituximab plus chlorambucil for six 28-day cycles [93]. The main study followed a safety run-in designed to ensure that chlorambucil-containing chemoimmunotherapy regimens were safe in recruited older patients with comorbidities [94]. IRRs and neutropenia were identified as potential risks during the run-in, but none of the specified stopping criteria were met, and the main study was opened for randomization in April 2010.

Both combinations increased PFS significantly over chlorambucil monotherapy; obinutuzumab plus chlorambucil, but not rituximab plus chlorambucil, prolonged OS significantly (Table 3) [95]. Obinutuzumab plus chlorambucil also conferred longer PFS (Table 3) $[93,95]$ and a higher CR rate (21\% vs. $7 \%)$ [93] than rituximab plus chlorambucil, and it was associated with significantly and substantially increased time to next treatment (Table 3) [95].

AEs were reported at higher frequencies with obinutuzumab plus chlorambucil than with either of the other two treatments, but toxicities were manageable and the risk of infection was not increased over rituximab plus chlorambucil or chlorambucil alone. Among patients for whom MRD data were available, the proportion who were MRD-negative in bone marrow and peripheral blood at the end of treatment was markedly higher with obinutuzumab plus chlorambucil than with rituximab plus chlorambucil treatment (bone marrow, $18 \%$ vs. 3\%; peripheral blood, $36 \%$ vs. 3\%) [96]. MRD in peripheral blood at the end of treatment was an independent prognostic factor for both PFS (HR 5.29; 95\% CI 3.48-8.04; $p<0.001$ ) and OS (HR 3.04; 95\% CI 1.53-6.03; $p=0.002)$.

The GREEN study (NCT01905943) is an ongoing, open-label, multicenter, phase IIIb study in patients with previously untreated or relapsed/refractory CLL receiving obinutuzumab alone or in combination with chemotherapy (bendamustine, FC, or chlorambucil). One of the objectives of this study is to investigate the potential of alternative obinutuzumab administration protocols for reducing IRRs. Emerging results suggest that the safety profile of obinutuzumab plus bendamustine is manageable if the appropriate measures are taken (e.g., monitoring at-risk patients for tumor lysis syndrome after the first infusion) $[97,98]$. 


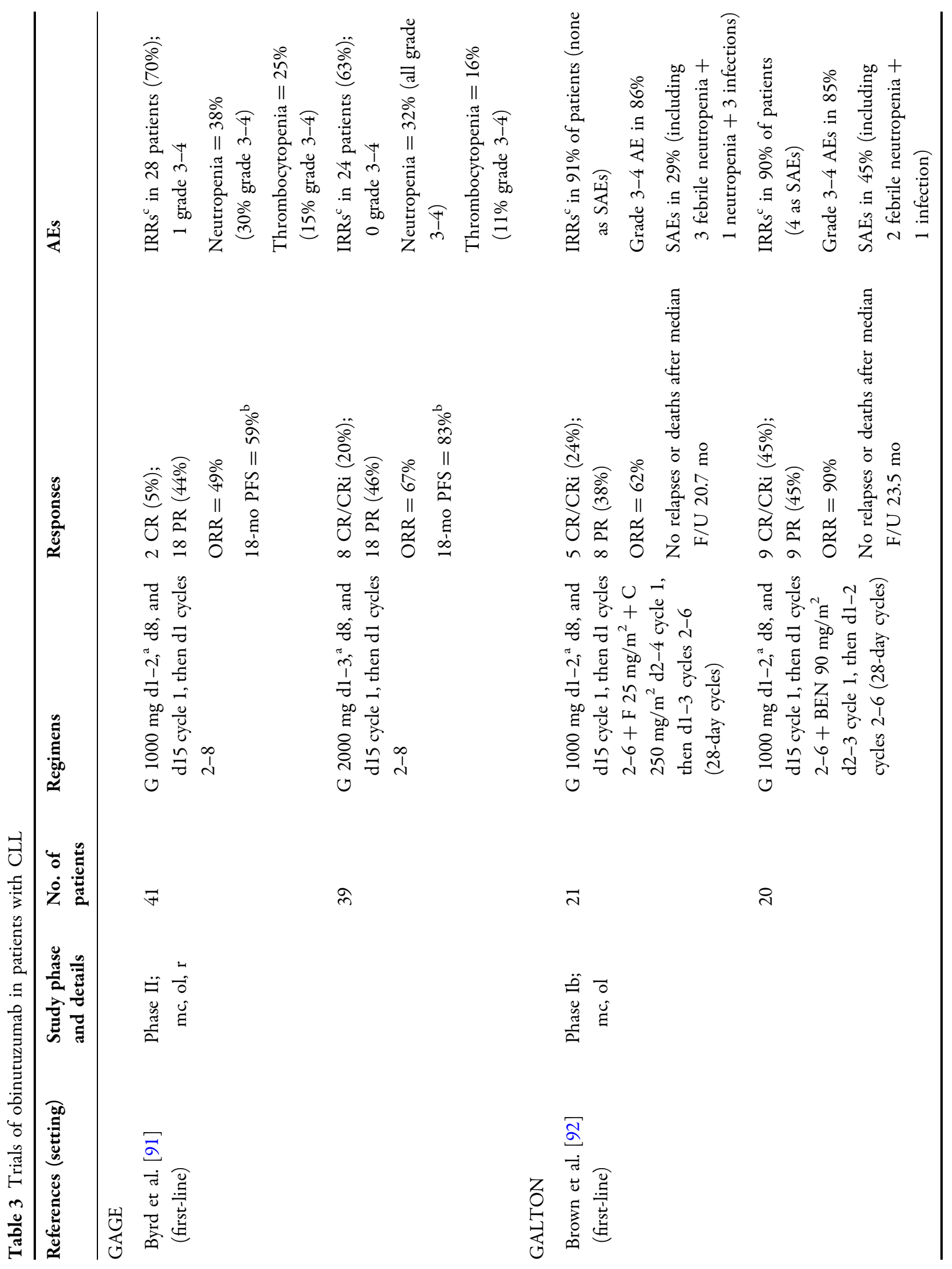




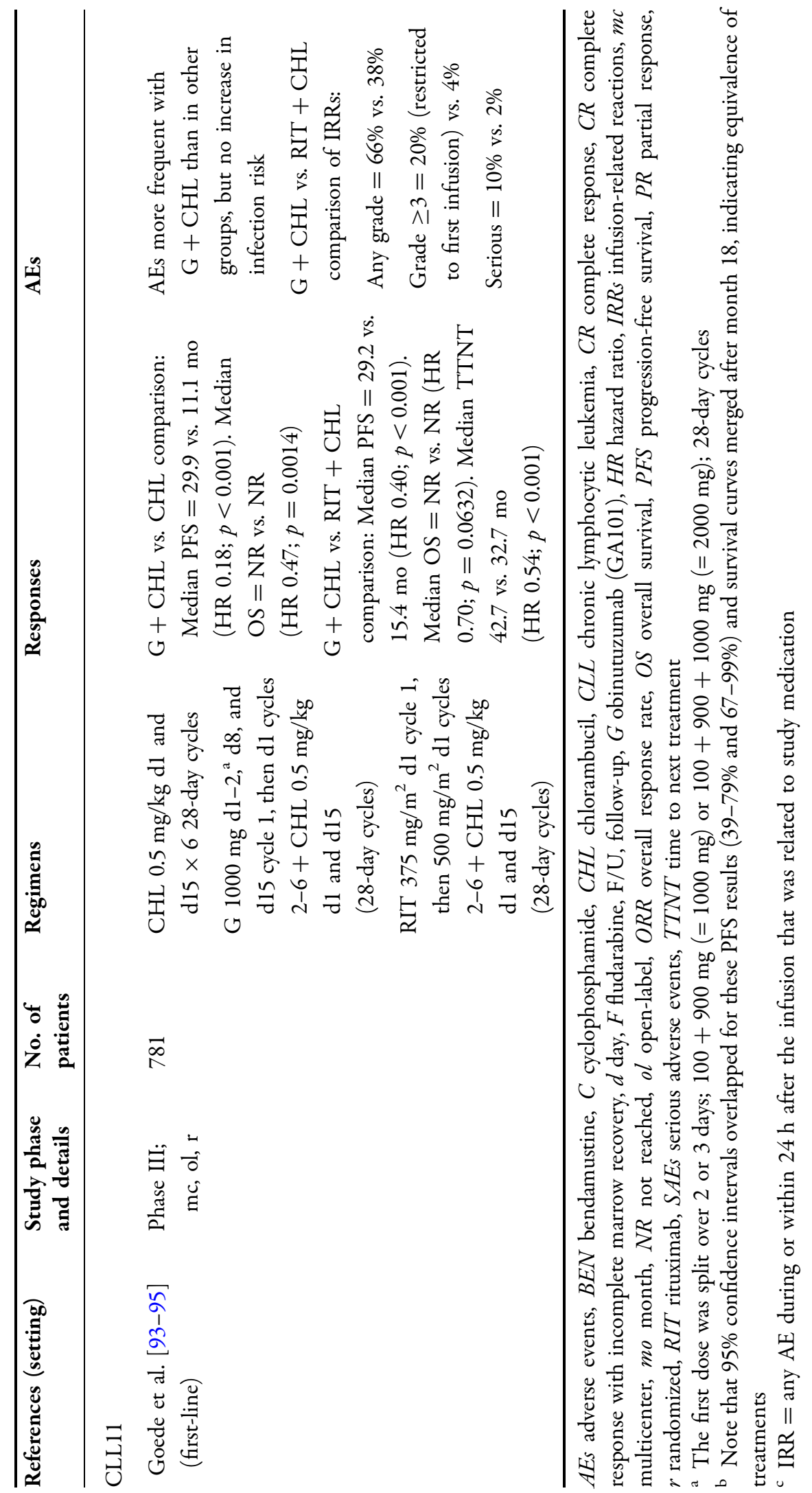




\section{DOSE RATIONALE}

In all phase III trials, obinutuzumab was, or is being, administered at the aforementioned rationally optimized 1000-mg flat-dose schedule [80], compared with the standard, approved $375 \mathrm{mg} / \mathrm{m}^{2}$ dose of rituximab in iNHL/DLBCL or $500 \mathrm{mg} / \mathrm{m}^{2}$ in CLL. A comparison of equal doses of the two mAbs was not feasible in the phase III trials because of the requirement for a third treatment arm and the associated need to recruit many additional patients. However, while there is controversy over whether the approved dose of rituximab is optimal, or which patients may benefit from a higher dose [99], there is no clinical evidence proving that increasing the dose of rituximab leads to better long-term outcomes for patients [100, 101]. Furthermore, differences between the obinutuzumab and rituximab arms in the CLL11 trial in terms of the kinetics of peripheral B-cell depletion [102] and achievement of MRD negativity [96] imply that the distinct biology of the two mAbs has a greater influence on the clinical results than the different dose schedules, and as such, increasing the dose of rituximab would be unlikely to result in comparable outcomes to obinutuzumab.

\section{SHORTER DURATION OF INFUSION}

Reducing the duration of infusion for intravenous drugs has potential advantages in terms of patient and physician burden. Long infusion times and frequent infusion rate changes result in lengthy observation times, increased nursing and administration staff workloads, and inconvenience to patients [103].

A notable early example of research to balance the benefits of shorter duration of infusion (SDI) against the potentially increased risk of hypersensitivity reactions was the effort made in the 1990s to reduce paclitaxel infusion times from 24 to $3 \mathrm{~h}$ [104]. Since then, SDI with rituximab has been investigated in 351 patients with rheumatoid arthritis in the RATE-RA study (NCT01382940): there was no increase in the rate or severity of IRRs when infusion times were reduced from the standard 4.25 to $2 \mathrm{~h}$ [103]. SDI with rituximab was subsequently assessed in a phase III study in 425 patients with DLBCL or FL [105]. Reduction of the infusion time from a median of $240 \mathrm{~min}$ to a fixed time of 90 min was found to be feasible in patients who tolerated an initial infusion at the longer standard rate. These observations led to an increase in the recommended infusion rate for rituximab to a maximum of $400 \mathrm{mg} / \mathrm{h}$ [106] and to the investigation of SDIs in patients receiving obinutuzumab. In the studies in which SDI was investigated, patients received obinutuzumab at the regular infusion rate for the first cycle to establish safety (i.e., no IRRs of grade $\geq 3$ ) and activity (lymphocyte count $\leq 5000 / \mu \mathrm{l})$, after which SDI could be started from cycle 2 .

\section{GATHER}

The safety of SDI of obinutuzumab after cycle 1 was evaluated in the GATHER study (first-line advanced DLBCL) (Table 2) [85]. After treatment at the regular infusion rate was deemed safe, based on 20 patients who received the standard-rate infusion with G-CHOP therapy, patients who met the SDI inclusion criteria (who had not experienced a serious and/or grade $\geq 3$ IRR and had a lymphocyte count $\leq 5000 / \mu \mathrm{l}$ ) were treated at the SDI rate. Initially three patients in the cohort received obinutuzumab over $120 \mathrm{~min}$; if none of the three experienced IRRs of grade $\geq 3$, the 90-min infusion was then tested for the 
remaining patients. Overall, 51 of 80 patients (64\%) experienced IRRs, most of which were of grade 1-2 in severity and reported in cycle 1 . There were no IRRs of grade $\geq 3$ with the 90-min infusion [85].

\section{GATS Study (JapicCTI-152848)}

SDI with obinutuzumab in combination with chemotherapy (CHOP) is also being investigated in a phase II study in Japanese patients (Table 2) receiving first-line therapy for various types of CD20-positive B-cell NHL. In cycle 1 , all patients receive obinutuzumab $1000 \mathrm{mg}$ on day 1 over $4.25 \mathrm{~h}$ and on days 8 and 15 over $3.25 \mathrm{~h}$. Patients who meet the criteria for SDI (no serious and/or grade $\geq 3$ IRRs in the first three infusions and lymphocyte count $\leq 5000 / \mu \mathrm{l}$ prior to SDI) receive their next obinutuzumab infusion over $1.5 \mathrm{~h}$. The primary objectives of the study are to evaluate infusion tolerability, pharmacokinetics, and change in cytokine levels over time.

\section{FUTURE PERSPECTIVES}

Since the introduction of rituximab in 1997, great advances have been made in the management of lymphoma and lymphocytic leukemia of B-cell origin. The formulation of truly novel treatment paradigms has been made possible, and multiple avenues of research are now being explored to find new and more effective ways of applying targeted therapy to maximize tumor responses and OS, while minimizing AEs and patient discomfort. This includes the expansion of combination therapy choices to include multiple targeted agents with the potential for reducing reliance on chemotherapy. Chemotherapy-free combinations have the potential to further optimize effector functions, and anti-CD20 therapy can reasonably be considered as a base treatment option. Anti-CD20 therapy holds great promise for the future management of B-cell NHL, and several novel entities are under development.

Several trials are now evaluating obinutuzumab in combination with other targeted therapies in B-cell NHL. The phase Ib/ II GALEN study (NCT01582776) is evaluating obinutuzumab in combination with lenalidomide in relapsed/refractory and first-line FL patients and in patients with aggressive B-cell lymphomas (DLBCL and $\mathrm{MCL})$. Phase Ib data from this study have already shown that the combination is effective, with an objective response in 13 of 19 evaluable patients, including 7 CRs [107]. Other similar studies include the GO27834 (ROMULUS) study (NCT01691898), a phase II study in which combinations of obinutuzumab or rituximab with the anti-CD79b antibody-drug conjugate, polatuzumab vedotin, are being tested in patients with relapsed/refractory B-cell NHL. In addition, the phase II GO29365 study (NCT02257567) is evaluating polatuzumab vedotin in combination with rituximab or obinutuzumab and bendamustine in patients with relapsed/ refractory FL or DLBCL. The novel oral BCL-2 inhibitor, GDC-0199 (ABT-199; venetoclax), is being investigated in a phase I/II study (GO27878; NCT02055820) in combination with rituximab or obinutuzumab plus $\mathrm{CHOP}$ in patients with B-cell NHL, while the phase I GO29383 study (NCT02220842) is assessing obinutuzumab in combination with atezolizumab (MPDL3280A) in relapsed/ refractory FL and DLBCL. Atezolizumab is a fully humanized antibody that acts against the protein ligand PD-L1.

There are also two phase III studies ongoing in first-line CLL. The CLL14 study (NCT02242942) will compare the efficacy and safety of a combined regimen of obinutuzumab 
and venetoclax versus obinutuzumab plus chlorambucil in patients with CLL and coexisting medical conditions. The anticipated time on study treatment is approximately 1 year, with a follow-up period of up to 5 years. The second study is evaluating the combination of obinutuzumab and ibrutinib, a Bruton's tyrosine kinase inhibitor, compared with obinutuzumab plus chlorambucil in patients with CLL or small lymphocytic leukemia (NCT02264574). Follow-up is planned for up to 3 years.

Other open-label, multicenter phase Ib/II studies in which obinutuzumab is being used in combination with other investigational agents include atezolizumab plus obinutuzumab and CHOP or bendamustine in first-line FL and DLBCL (NCT02596971); atezolizumab plus obinutuzumab and lenalidomide in relapsed/refractory FL (NCT02631577); atezolizumab plus obinutuzumab and polatuzumab vedotin in relapsed/refractory FL and DLBCL (NCT02729896); obinutuzumab plus polatuzumab vedotin and lenalidomide in relapsed/refractory FL and DLBCL (NCT02600897); obinutuzumab plus polatuzumab vedotin and venetoclax in relapsed/refractory FL and DLBCL (NCT02611323); and obinutuzumab plus idasanutlin (an MDM2 antagonist) in relapsed/ refractory FL and DLBCL (NCT02624986). A phase Ib study examining the combination of obinutuzumab plus idasanutlin and venetoclax is also planned.

A large number of other molecular targets are being explored for their therapeutic potential in hematologic malignancies; these are beyond the scope of this review and are discussed elsewhere [45]. However, novel approaches designed to promote targeting of tumors by T-cells are also being investigated. Bispecific
T-cell engagers (BiTE) contain the variable domains of two antibodies joined together, one of which binds CD19, while the other binds T-cell CD3 [108]. The creation of a CD19/ CD3 complex brings tumor and T-cells together in close proximity, which in turn activates the T-cell and causes it to destroy the tumor cell via perforin-mediated apoptosis [45]. Other researchers are exploring the potential of anti-CD20/CD3 bispecific antibodies to overcome problems of short half-life in vivo, structural instability, and poor solubility of anti-CD19 BiTEs [109-111]. Another approach is modification with chimeric antigen receptors (CARs) of T-cells to confer tumor-specific cytotoxicity [112]. Preclinical and clinical studies of CD19 CAR-T-cells have shown encouraging results in a variety of cancers, particularly B-cell hematologic malignancies [113].

There is also a need to explore the potential applicability of surrogate endpoints in hematologic malignancies to help direct therapy and to expedite the conduct of clinical trials. Positron emission tomography using 18-fluoro-2-deoxyglucose has become a standard clinical tool for staging and response assessment in aggressive lymphomas, and results from a number of studies indicate its potential utility in predicting outcomes in patients with DLBCL and FL [114, 115]. Another approach is to measure MRD (as described above for GADOLIN and CLL11) $[88,96]$, which can be used for evaluation of treatment effectiveness, risk stratification, and long-term outcome prediction. While multicolor flow cytometry and polymerase chain reaction-based methods are currently the two most commonly used techniques for assessing MRD, next-generation sequencing is likely to be more widely employed in the future [116]. 
PFS is the standard endpoint for assessing new drugs in first-line $\mathrm{FL}$, but the increasing efficacy of new treatments and the indolent nature of FL demand extended patient follow-up in clinical trials [117]. There is therefore interest in whether CR at 30 months (CR30) could accurately predict likely treatment effects on PFS. A recent meta-analysis of data from 13 randomized, first-line trials in 3837 patients with individual patient data has supported this hypothesis [117]: an absolute improvement in CR30 of $\geq 10 \%$ over a control CR30 of $50 \%$ predicted a significant improvement in PFS. This suggests that CR30 has utility as a surrogate for PFS in first-line FL trials and supports its use to facilitate treatment development.

In conclusion, researchers are continuing their efforts to develop increasingly efficient therapies that provide long treatment-free periods for patients with relapsing or refractory disease, to identify the most effective combination therapies (notably triplet combinations) and, as discussed above, to develop chemotherapy-free regimens based on immunotherapy. The advances with obinutuzumab described here against the background of the many other therapeutic approaches that are underway are evidence of marked and significant progress in the development and application of immunotherapy against lymphocytic leukemia and B-cell lymphoma.

\section{ACKNOWLEDGEMENTS}

The article processing charges were funded by Chugai Pharmaceutical Co., Ltd., Chuo-ku, Tokyo, Japan. All named authors meet the International Committee of Medical Journal Editors (ICMJE) criteria for authorship for this manuscript, take responsibility for the integrity of the work as a whole, and have given final approval to the version to be published. All authors had full access to the data and take complete responsibility for the integrity of the data and accuracy of the data presentation. Third-party medical writing assistance, under the direction of KT, was provided by Christopher Dunn and Scott Malkin of Gardiner-Caldwell Communications, an Ashfield company, part of UDG Healthcare PLC, and was funded by Chugai Pharmaceutical Co., Ltd. We thank the patients, their family members, investigators, nurses, and clinical research coordinators who have contributed to various clinical trials of obinutuzumab.

Disclosures. Kensei Tobinai has received research grants from Chugai, Roche, Zenyaku Kogyo, and Novartis. Christian Klein is an employee at F. Hoffmann-La Roche Ltd. and owns stock in F. Hoffmann-La Roche Ltd. Naoko Oya is an employee at Chugai Pharmaceutical Co., Ltd. Günter Fingerle-Rowson is an employee at F. Hoffmann-La Roche Ltd. and owns stock in F. Hoffmann-La Roche Ltd.

Compliance with Ethics Guidelines. This article does not contain any new studies with human or animal subjects performed by any of the authors.

Data Availability. Data sharing is not applicable to this article as no datasets were generated or analyzed in this review.

Open Access. This article is distributed under the terms of the Creative Commons Attribution-NonCommercial 4.0 International License (http://creativecommons.org/licenses/ 
by-nc/4.0/), which permits any noncommercial use, distribution, and reproduction in any medium, provided you give appropriate credit to the original author(s) and the source, provide a link to the Creative Commons license, and indicate if changes were made.

\section{REFERENCES}

1. American Cancer Society. What is chronic lymphocytic leukemia? 2016. http://www.cancer. org/cancer/leukemia-chroniclymphocyticcll/detailed guide/leukemia-chronic-lymphocytic-what-is-cll. Accessed 20 July 2016.

2. Eichhorst B, Robak T, Montserrat E, et al. Chronic lymphocytic leukaemia: ESMO Clinical Practice Guidelines for diagnosis, treatment and follow-up. Ann Oncol. 2015;26(suppl 5):v78-84.

3. Swerdlow SH, Campo E, Harris NL, et al., editors. WHO classification of tumours of haematopoietic and lymphoid tissue (IARC WHO classification of tumours). 4th ed. Geneva: World Health Organization; 2008.

4. Swerdlow SH, Campo E, Pileri SA, et al. The 2016 revision of the World Health Organization classification of lymphoid neoplasms. Blood. 2016;127:2375-90.

5. Lymphoma Research Foundation. About lymphoma. 2016. http://www.lymphoma.org/site/ pp.asp?c=bkLTKaOQLmK8E\&b=6299689. Accessed 20 July 2016.

6. Zappasodi R, de Braud F, Di Nicola M. Lymphoma immunotherapy: current status. Front Immunol. 2015;6:448.

7. Dreyling M, Ghielmini M, Marcus R, et al. Newly diagnosed and relapsed follicular lymphoma: ESMO Clinical Practice Guidelines for diagnosis, treatment and follow-up. Ann Oncol. 2014;25(suppl 3):iii76-82.

8. Jaglowski SM, Linden E, Termuhlen AM, Flynn JM. Lymphoma in adolescents and young adults. Semin Oncol. 2009;36:381-418.

9. Smith SM. Dissecting follicular lymphoma: high versus low risk. Hematol Am Soc Hematol Educ Progr. 2013;2013:561-7.

10. Salles G, Seymour JF, Offner F, et al. Rituximab maintenance for 2 years in patients with high tumour burden follicular lymphoma responding to rituximab plus chemotherapy (PRIMA): a phase 3, randomised controlled trial. Lancet. 2011;377:42-51.

11. Rummel MJ, Niederle N, Maschmeyer G, et al. Bendamustine plus rituximab versus CHOP plus rituximab as first-line treatment for patients with indolent and mantle-cell lymphomas: an open-label, multicentre, randomised, phase 3 non-inferiority trial. Lancet. 2013;381:1203-10.

12. Chao MP. Treatment challenges in the management of relapsed or refractory non-Hodgkin's lymphoma-novel and emerging therapies. Cancer Manag Res. 2013;5:251-69.

13. Tilly H, Gomes da Silva M, Vitolo U, et al. Diffuse large B-cell lymphoma (DLBCL): ESMO Clinical Practice Guidelines for diagnosis, treatment and follow-up. Ann Oncol. 2015;26(suppl 5):v116-25.

14. Ghielmini M, Vitolo U, Kimby E, et al. ESMO Guidelines consensus conference on malignant lymphoma 2011 part 1: diffuse large B-cell lymphoma (DLBCL), follicular lymphoma (FL) and chronic lymphocytic leukemia (CLL). Ann Oncol. 2012;24:561-76.

15. Vinjamaram S. Non-Hodgkin lymphoma treatment \& management: approach considerations, management of indolent NHL, management of aggressive NHL. 2016. http://emedicine.medscape. com/article/203399-treatment\#d7. Accessed 20 July 2016.

16. Bierman PJ, Vose JM, Anderson JR, et al. High-dose therapy with autologous hematopoietic rescue for follicular low-grade non-Hodgkin's lymphoma. J Clin Oncol. 1997;15:445-50.

17. Maloney DG, Grillo-Lopez AJ, White CA, et al. IDEC-C2B8 (Rituximab) anti-CD20 monoclonal antibody therapy in patients with relapsed low-grade non-Hodgkin's lymphoma. Blood. 1997;90:2188-95.

18. McLaughlin P, Grillo-Lopez AJ, Link BK, et al. Rituximab chimeric anti-CD20 monoclonal antibody therapy for relapsed indolent lymphoma: half of patients respond to a four-dose treatment program. J Clin Oncol. 1998;16:2825-33.

19. Hainsworth JD. Monoclonal antibody therapy in lymphoid malignancies. Oncologist. 2000;5:376-84.

20. Davis TA, Czerwinski DK, Levy R. Therapy of B-cell lymphoma with anti-CD20 antibodies can result in the loss of CD20 antigen expression. Clin Cancer Res. 1999;5:611-5. 
21. Bachy E, Salles G. Are we nearing an era of chemotherapy-free management of indolent lymphoma? Clin Cancer Res. 2014;20:5226-39.

22. Tan D, Horning SJ, Hoppe RT, et al. Improvements in observed and relative survival in follicular grade 1-2 lymphoma during 4 decades: the Stanford University experience. Blood. 2013;122:981-7.

23. Lim SH, Levy R. Translational medicine in action: anti-CD20 therapy in lymphoma. J Immunol. 2014;193:1519-24.

24. Coiffier B, Thieblemont C, Van Den Neste E, et al. Long-term outcome of patients in the LNH-98.5 trial, the first randomized study comparing rituximab-CHOP to standard CHOP chemotherapy in DLBCL patients: a study by the Groupe d'Etudes des Lymphomes de l'Adulte. Blood. 2010;116:2040-5.

25. Pfreundschuh M, Trumper L, Osterborg A, et al. CHOP-like chemotherapy plus rituximab versus CHOP-like chemotherapy alone in young patients with good-prognosis diffuse large-B-cell lymphoma: a randomised controlled trial by the MabThera International Trial (MInT) Group. Lancet Oncol. 2006;7:379-91.

26. Hallek M, Fischer K, Fingerle-Rowson G, et al. Addition of rituximab to fludarabine and cyclophosphamide in patients with chronic lymphocytic leukaemia: a randomised, open-label, phase 3 trial. Lancet. 2010;376:1164-74.

27. Bachy E, Brice P, Delarue R, et al. Long-term follow-up of patients with newly diagnosed follicular lymphoma in the prerituximab era: effect of response quality on survival-a study from the Groupe d'Etude des Lymphomes de l'Adulte. J Clin Oncol. 2010;28:822-9.

28. Cohen JB, Flowers CR. Optimal disease surveillance strategies in non-Hodgkin lymphoma. Hematol Am Soc Hematol Educ Progr. 2014;2014:481-7.

29. Alduaij W, Illidge TM. The future of anti-CD20 monoclonal antibodies: are we making progress? Blood. 2011;117:2993-3001.

30. Owen CJ, Stewart DA. Obinutuzumab for the treatment of patients with previously untreated chronic lymphocytic leukemia: overview and perspective. Ther Adv Hematol. 2015;6:161-70.

31. Beum PV, Lindorfer MA, Taylor RP. Within peripheral blood mononuclear cells, antibody-dependent cellular cytotoxicity of rituximab-opsonized Daudi cells is promoted by NK cells and inhibited by monocytes due to shaving. J Immunol. 2008;181:2916-24.
32. Beum PV, Peek EM, Lindorfer MA, et al. Loss of CD20 and bound CD20 antibody from opsonized B cells occurs more rapidly because of trogocytosis mediated by Fc receptor-expressing effector cells than direct internalization by the $B$ cells. J Immunol. 2011;187:3438-47.

33. Taylor RP, Lindorfer MA. Fc $\gamma$-receptor-mediated trogocytosis impacts mAb-based therapies: historical precedence and recent developments. Blood. 2015;125:762-6.

34. Dransfield I. Inhibitory Fc $\gamma$ RIIb and CD20 internalization. Blood. 2014;123:606-7.

35. Vaughan AT, Iriyama C, Beers SA, et al. Inhibitory Fc $\gamma$ RIIb (CD32b) becomes activated by therapeutic $\mathrm{mAb}$ in both cis and trans and drives internalization according to antibody specificity. Blood. 2014;123:669-77.

36. Tipton TR, Roghanian A, Oldham RJ, et al. Antigenic modulation limits the effector cell mechanisms employed by type I anti-CD20 monoclonal antibodies. Blood. 2015;125:1901-9.

37. Lim SH, Vaughan AT, Ashton-Key M, et al. Fc gamma receptor IIb on target B cells promotes rituximab internalization and reduces clinical efficacy. Blood. 2011;118:2530-40.

38. Goede V, Klein C, Stilgenbauer S. Obinutuzumab (GA101) for the treatment of chronic lymphocytic leukemia and other B-cell non-Hodgkin's lymphomas: a glycoengineered type II CD20 antibody. Oncol Res Treat. 2015;38:185-92.

39. Mössner E, Brünker P, Moser S, et al. Increasing the efficacy of CD20 antibody therapy through the engineering of a new type II anti-CD20 antibody with enhanced direct and immune effector cell-mediated B-cell cytotoxicity. Blood. 2010;115:4393-402.

40. Niederfellner G, Lammens A, Mundigl O, et al. Epitope characterization and crystal structure of GA101 provide insights into the molecular basis for type I/II distinction of CD20 antibodies. Blood. 2011;118:358-67.

41. Goede V, Fischer K, Humphrey K, et al. Obinutuzumab (GA101) plus chlorambucil (Clb) or rituximab (R) plus $\mathrm{Clb}$ versus $\mathrm{Clb}$ alone in patients with chronic lymphocytic leukemia (CLL) and preexisting medical conditions (comorbidities): final stage 1 results of the CLL11 (BO21004) phase III trial. J Clin Oncol. 2013;31(suppl) (abstract 7004).

42. Klein C, Lammens A, Schafer W, et al. Epitope interactions of monoclonal antibodies targeting 
CD20 and their relationship to functional properties. MAbs. 2013;5:22-33.

43. Herter S, Herting F, Mundigl O, et al. Preclinical activity of the type II CD20 antibody GA101 (obinutuzumab) compared with rituximab and ofatumumab in vitro and in xenograft models. Mol Cancer Ther. 2013;12:2031-42.

44. Herter S, Birk MC, Klein C, Gerdes C, Umana P, Bacac M. Glycoengineering of therapeutic antibodies enhances monocyte/macrophage-mediated phagocytosis and cytotoxicity. J Immunol. 2014;192:2252-60.

45. Suresh T, Lee LX, Joshi J, Barta SK. New antibody approaches to lymphoma therapy. J Hematol Oncol. 2014;7:58.

46. Teeling JL, Mackus WJ, Wiegman LJ, et al. The biological activity of human CD20 monoclonal antibodies is linked to unique epitopes on CD20. J Immunol. 2006;177:362-71.

47. Golay G, Bologna L, André PA, et al. Possible misinterpretation of the mode of action of therapeutic antibodies in vitro: homotypic adhesion and flow cytometry result in artefactual direct cell death. Blood. 2010;116:3372-3.

48. Honeychurch J, Alduaij W, Azizyan M, et al. Antibody-induced nonapoptotic cell death in human lymphoma and leukemia cells is mediated through a novel reactive oxygen species-dependent pathway. Blood. 2012;119:3523-33.

49. Cheadle EJ, Sidon L, Dovedi SJ, et al. The induction of immunogenic cell death by type II anti-CD20 monoclonal antibodies has mechanistic differences compared with type I rituximab. Br J Haematol. 2013;162:842-5.

50. Rafiq S, Butchar JP, Cheney C, et al. Comparative assessment of clinically utilized CD20-directed antibodies in chronic lymphocytic leukemia cells reveals divergent NK cell, monocyte, and macrophage properties. J Immunol. 2013;190:2702-11.

51. Alduaij W, Ivanov A, Honeychurch J, et al. Novel type II anti-CD20 monoclonal antibody (GA101) evokes homotypic adhesion and actin-dependent, lysosome-mediated cell death in B-cell malignancies. Blood. 2011;117:4519-29.

52. Reslan L, Dalle S, Herveau S, et al. Apoptotic induction by anti-CD20 antibodies in chronic lymphocytic leukemia: comparison of rituximab and obinutuzumab. Leuk Lymphoma. 2014;55:188-90.

53. Dalle S, Reslan L, Besseyre de Horts T, et al. Preclinical studies on the mechanism of action and the anti-lymphoma activity of the novel anti-CD20 antibody GA101. Mol Cancer Ther. 2011;10:178-85.

54. Cragg MS, Alduaij W, Klein C, et al. Response: novel lysosomal-dependent cell death following homotypic adhesion occurs within cell aggregates. Blood. 2010;116:3373-4.

55. Jak M, van Bochove GG, Reits EA, et al. CD40 stimulation sensitizes CLL cells to lysosomal cell death induction by type II anti-CD20 mAb GA101. Blood. 2011;118:5178-88.

56. Nagy ZA, Hubner B, Löhning C, et al. Fully human, HLA-DR-specific monoclonal antibodies efficiently induce programmed death of malignant lymphoid cells. Nat Med. 2002;8:801-7.

57. Heider $\mathrm{KH}$, Kiefer $\mathrm{K}$, Zenz $\mathrm{T}$, et al. A novel Fc-engineered monoclonal antibody to CD37 with enhanced ADCC and high proapoptotic activity for treatment of B-cell malignancies. Blood. 2011;118:4159-68.

58. Patz M, Isaeva $\mathrm{P}$, Forcob $\mathrm{N}$, et al. Comparison of the in vitro effects of the anti-CD20 antibodies rituximab and GA101 on chronic lymphocytic leukaemia cells. Br J Haematol. 2011;152:295-306.

59. Bologna L, Gotti E, Manganini M, et al. Mechanism of action of type II, glycoengineered, anti-CD20 monoclonal antibody GA101 in B-chronic lymphocytic leukemia whole blood assays in comparison with rituximab and alemtuzumab. J Immunol. 2011;186:3762-9.

60. Laprevotte E, Ysebaert L, Klein C, et al. Endogenous IL-8 acts as a CD16 co-activator for natural killer-mediated anti-CD20 B cell depletion in chronic lymphocytic leukemia. Leuk Res. 2013;37:440-6.

61. Laprevotte E, Voisin G, Ysebaert L, et al. Recombinant human IL-15 trans presentation by $\mathrm{B}$ leukemic cells from chronic lymphocytic leukemia induces autologous NK cell proliferation leading to improved anti-CD20 immunotherapy. J Immunol. 2013;191:3634-40.

62. Wang SY, Weiner G. Complement and cellular cytotoxicity in antibody therapy of cancer. Expert Opin Biol Ther. 2008;8:759-68.

63. Kern DJ, James BR, Blackwell S, Gassner C, Klein C, Weiner GJ. GA101 induces NK-cell activation and antibody-dependent cellular cytotoxicity more effectively than rituximab when complement is present. Leuk Lymphoma. 2013;54:2500-5.

64. Grandjean CL, Montalvao F, Celli S, et al. Intravital imaging reveals improved Kupffer cell-mediated 
phagocytosis as a mode of action of glycoengineered anti-CD20 antibodies. Sci Rep. 2016;6:34382.

65. Terszowski G, Klein C, Stern M. KIR/HLA interactions negatively affect rituximab- but not GA101 (obinutuzumab)-induced antibody-dependent cellular cytotoxicity. J Immunol. 2014;192:5618-24.

66. Terszowski G, Crompton G, Goede V, et al. Association between KIR-HLA genotype and outcome in the CLL11 study of chronic lymphocytic leukemia patients receiving chlorambucil alone or in combination with rituximab or obinutuzumab. Haematologica. 2016;101(s1):P216.

67. Vaughan AT, Cragg MS, Beers SA. Antibody modulation: limiting the efficacy of therapeutic antibodies. Pharmacol Res. 2015;99:269-75.

68. Ysebaert L, Laprevotte E, Klein C, Quillet-Mary A. Obinutuzumab (GA101) is highly effective against chronic lymphocytic leukemia cells in ex vivo B-cell depletion irrespective of high-risk prognostic markers. Blood Cancer J. 2015;5:e367.

69. Herting F, Friess T, Bader S, et al. Enhanced anti-tumor activity of the glycoengineered type II CD20 antibody obinutuzumab (GA101) in combination with chemotherapy in xenograft models of human lymphoma. Leuk Lymphoma. 2014;55:2151-60.

70. Salles G, Morschhauser F, Lamy T, et al. Phase 1 study results of the type II glycoengineered humanized anti-CD20 monoclonal antibody obinutuzumab (GA101) in B-cell lymphoma patients. Blood. 2012;119:5126-32.

71. Salles GA, Morschhauser F, Solal-Celigny P, et al. Obinutuzumab (GA101) in patients with relapsed/ refractory indolent non-Hodgkin lymphoma: results from the phase II GAUGUIN study. J Clin Oncol. 2013;31:2920-6.

72. Morschhauser FA, Cartron G, Thieblemont C, et al. Obinutuzumab (GA101) monotherapy in relapsed/ refractory diffuse large B-cell lymphoma or mantle-cell lymphoma: results from the phase II GAUGUIN study. J Clin Oncol. 2013;31:2912-9.

73. Cartron G, de Guibert S, Dilhuydy MS, et al. Obinutuzumab (GA101) in relapsed/refractory chronic lymphocytic leukemia: final data from the phase 1/2 GAUGUIN study. Blood. 2014;124:2196-202.

74. Sehn LH, Goy A, Offner FC, et al. Randomized phase II trial comparing obinutuzumab (GA101) with rituximab in patients with relapsed
CD20 + indolent B-cell non-Hodgkin lymphoma: final analysis of the GAUSS study. J Clin Oncol. 2015;33:3467-74.

75. Ogura M, Tobinai K, Hatake K, et al. Phase I study of obinutuzumab (GA101) in Japanese patients with relapsed or refractory B-cell non-Hodgkin lymphoma. Cancer Sci. 2012;104:105-10.

76. Byrd JC, Murphy T, Howard RS, et al. Rituximab using a thrice weekly dosing schedule in B-cell chronic lymphocytic leukemia and small lymphocytic lymphoma demonstrates clinical activity and acceptable toxicity. J Clin Oncol. 2001;19:2153-64.

77. Byrd JC, Waselenko JK, Maneatis TJ, et al. Rituximab therapy in hematologic malignancy patients with circulating blood tumor cells: association with increased infusion-related side effects and rapid blood tumor clearance. J Clin Oncol. 1999;17:791-5.

78. Winkler U, Jensen M, Manzke O, Schulz H, Diehl V, Engert A. Cytokine-release syndrome in patients with B-cell chronic lymphocytic leukemia and high lymphocyte counts after treatment with an anti-CD20 monoclonal antibody (rituximab, IDEC-C2B8). Blood. 1999;94:2217-24.

79. Freeman CL, Morschhauser F, Sehn L, et al. Cytokine release in patients with CLL treated with obinutuzumab and possible relationship with infusion-related reactions. Blood. 2015;126:2646-9.

80. Cartron G, Hourcade-Potelleret F, Morschhauser F, et al. Rationale for optimal obinutuzumab/GA101 dosing regimen in B-cell non-Hodgkin lymphoma. Haematologica. 2016;101:226-34.

81. Gibiansky E, Gibiansky L, Carlile DJ, et al. Population pharmacokinetics of obinutuzumab (GA101) in chronic lymphocytic leukemia (CLL) and non-Hodgkin's lymphoma and exposure-response in CLL. CPT Pharmacometr Syst Pharmacol. 2014;3:e144.

82. Sehn LH, Assouline SE, Stewart DA, et al. A phase 1 study of obinutuzumab induction followed by 2 years of maintenance in patients with relapsed CD20-positive B-cell malignancies. Blood. 2012;119:5118-25.

83. Radford J, Davies A, Cartron G, et al. Obinutuzumab (GA101) plus CHOP or FC in relapsed/refractory follicular lymphoma: results of the GAUDI study (BO21000). Blood. 2013;122:1137-43.

84. Dyer MJS, Grigg AP, Gonzàlez Dìaz $M$, et al. Obinutuzumab (GA101) in combination with CHOP (cyclophosphamide, doxorubicin, vincristine and prednisone) or bendamustine for 
the first-line treatment of follicular non-Hodgkin lymphoma: final results from the maintenance phase of the phase Ib GAUDI study. Blood. 2014;124:1743.

85. Zelenetz AD, Mobasher M, Costa LJ, et al. Safety and efficacy of obinutuzumab (GA101) plus CHOP chemotherapy in first-line advanced diffuse large B-cell lymphoma: results from the phase 2 GATHER study (GAO4915g). Blood. 2013;122: 1820.

86. Sehn LH, Chua N, Mayer J, et al. A randomised controlled trial of obinutuzumab plus bendamustine versus bendamustine alone in patients with rituximab-refractory indolent non-Hodgkin lymphoma: primary results of the GADOLIN study. Lancet Oncol. 2016;17:1081-93.

87. Trněný $M$, Bouabdallah $K$, Dueck $G$, et al. Obinutuzumab plus bendamustine versus bendamustine alone in patients with rituximab-refractory follicular lymphoma: results from the GADOLIN study. Haematologica. 2016;101(S1):S440.

88. Pott C, Belada D, Danesi N, et al. Analysis of minimal residual disease in follicular lymphoma patients in GADOLIN, a phase III study of obinutuzumab plus bendamustine versus bendamustine in relapsed/refractory indolent non-Hodgkin lymphoma. Blood. 2015;126:3978.

89. Cheson BD, Trask PC, Gribben J, et al. Primary results of the health-related quality of life assessment from the phase III GADOLIN study of obinutuzumab plus bendamustine compared with bendamustine alone in patients with rituximab-refractory, indolent non-Hodgkin lymphoma. Blood. 2015;126:1532.

90. F. Hoffman-La Roche Ltd. Roche's GAZYVA/ GAZYVARO showed superior progression-free survival compared to MabThera/Rituxan in people with previously untreated follicular lymphoma [press release]. Basel, Switzerland. 2016. http:// www.roche.com/media/store/releases/med-cor-201605-27.htm. Accessed 20 July 2016.

91. Byrd JC, Flynn JM, Kipps TJ, et al. Randomized phase 2 study of obinutuzumab monotherapy in symptomatic, previously untreated chronic lymphocytic leukemia. Blood. 2016;127:79-86.

92. Brown JR, O'Brien S, Kingsley CD, et al. Obinutuzumab plus fludarabine/ cyclophosphamide or bendamustine in the initial therapy of CLL patients: the phase $1 \mathrm{~b}$ GALTON trial. Blood. 2015;125:2779-85.

93. Goede V, Fischer K, Busch R, et al. Obinutuzumab plus chlorambucil in patients with CLL and coexisting conditions. N Engl J Med. 2014;370:1101-10.

94. Goede V, Fischer K, Busch R, et al. Chemoimmunotherapy with GA101 plus chlorambucil in patients with chronic lymphocytic leukemia and comorbidity: results of the CLL11 (BO21004) safety run-in. Leukemia. 2013;27:1172-4.

95. Goede V, Fischer K, Engelke A, et al. Obinutuzumab as frontline treatment of chronic lymphocytic leukemia: updated results of the CLL11 study. Leukemia. 2015;29:1602-4.

96. Ritgen M, Langerak A, Goede V, et al. Quantitative MRD is prognostic for progression-free \& overall survival in elderly patients receiving chlorambucil alone or with obinutuzumab/rituximab: a prospective analysis of the GCLLSG CLL11 study. Haematologica. 2016;101(S1):S428.

97. Bosch F, Illmer T, Turgut M, et al. Preliminary safety results from the phase IIIb GREEN study of obinutuzumab (GA101) alone or in combination with chemotherapy for previously untreated or relapsed/refractory chronic lymphocytic leukemia (CLL). Blood. 2014;124:3345.

98. Stilgenbauer S, Ilhan O, Woszczyk D, et al. Safety and efficacy of obinutuzumab plus bendamustine in previously untreated patients with chronic lymphocytic leukemia: subgroup analysis of the GREEN study. Blood. 2015;126:493.

99. Golay J, Semenzato G, Rambaldi A, et al. Lessons for the clinic from rituximab pharmacokinetics and pharmacodynamics. MAbs. 2013;5:826-37.

100. Lugtenburg PJ, de Nully Brown P, van der Holt B, et al. Randomized phase III study on the effect of early intensification of rituximab in combination with 2-weekly CHOP chemotherapy followed by rituximab or no maintenance in patients with diffuse large B-cell lymphoma: results from a HOVON-Nordic Lymphoma Group study. J Clin Oncol. 2016;34(suppl) (abstract 7504).

101. Coiffier B, Haioun C, Ketterer N, et al. Rituximab (anti-CD20 monoclonal antibody) for the treatment of patients with relapsing or refractory aggressive lymphoma: a multicenter phase II study. Blood. 1998;92:1927-32.

102. Goede V, Fischer K, Kreuzer K-A, et al. Kinetics of blood cell subpopulations during treatment with obinutuzumab (GA101) + chlorambucil (GClb), rituximab + chlorambucil (RClb) versus chlorambucil (Clb) alone in patients with chronic lymphocytic leukemia and coexisting medical conditions: stage 1 results of the CLL11 (BO21004) trial. 15th International Workshop on Chronic 
Lymphocytic Leukaemia (iwCLL). Abstracts. 2013;4:14

103. Pritchard $\mathrm{CH}$, Greenwald MW, Kremer JM, et al. Safety of infusing rituximab at a more rapid rate in patients with rheumatoid arthritis: results from the RATE-RA study. BMC Musculoskelet Disord. 2014;15:177.

104. Williams C, Collingwood M, Simera I, Grafton C. Short versus long duration infusions of paclitaxel for any adenocarcinoma. Cochrane Database Syst Rev. 2003:Cd003911.

105. Dakhil S, Hermann R, Schreeder MT, et al. Phase III safety study of rituximab administered as a 90-minute infusion in patients with previously untreated diffuse large B-cell and follicular lymphoma. Leuk Lymphoma. 2014;55:2335-40.

106. Genentech USA, Inc. Highlights of prescribing information. RITUXAN ${ }^{\circledR}$ (rituximab) injection, for intravenous use. April 2016. http://www.gene.com/ download/pdf/rituxan_prescribing.pdf. Accessed 20 July 2016.

107. Morschhauser F, Salles G, Le Gouill S, et al. A phase Ib study of obinutuzumab combined with lenalidomide for relapsed/refractory follicular B-cell lymphoma. Blood. 2014;124:4458.

108. Smits NC, Sentman CL. Bispecific T-cell engagers (BiTEs) as treatment of B-cell lymphoma. J Clin Oncol. 2016;34:1131-3.

109. Lu CY, Chen GJ, Tai PH, et al. Tetravalent anti-CD20/CD3 bispecific antibody for the treatment of B cell lymphoma. Biochem Biophys Res Commun. 2016;473:808-13.

110. Sun LL, Ellerman D, Mathieu M, et al. Anti-CD20/ CD3 $\mathrm{T}$ cell-dependent bispecific antibody for the treatment of B cell malignancies. Sci Transl Med. 2015;7:287ra70.
111. Smith EJ, Olson K, Haber LJ, et al. A novel, native-format bispecific antibody triggering T-cell killing of B-cells is robustly active in mouse tumor models and cynomolgus monkeys. Sci Rep. 2015;5:17943.

112. Zhang H, Ye ZL, Yuan ZG, Luo ZQ, Jin HJ, Qian QJ. New strategies for the treatment of solid tumors with CAR-T cells. Int J Biol Sci. 2016;12:718-29.

113. Maus MV, Grupp SA, Porter DL, June $\mathrm{CH}$. Antibody-modified $T$ cells: CARs take the front seat for hematologic malignancies. Blood. 2014;123:2625-35.

114. Coughlan M, Elstrom R. The use of FDG-PET in diffuse large $B$ cell lymphoma (DLBCL): predicting outcome following first line therapy. Cancer Imaging. 2015;14:34.

115. Trotman J, Luminari S, Boussetta S, et al. Prognostic value of PET-CT after first-line therapy in patients with follicular lymphoma: a pooled analysis of central scan review in three multicentre studies. Lancet Haematol. 2014;1:e17-27.

116. Hauwel M, Matthes T. Minimal residual disease monitoring: the new standard for treatment evaluation of haematological malignancies? Swiss Med Wkly. 2014;144:w13907.

117. Sargent DJ, Shi Q, De Bedout S, et al. Evaluation of complete response rate at 30 months (CR30) as a surrogate for progression-free survival (PFS) in first-line follicular lymphoma (FL) studies: results from the prospectively specified Follicular Lymphoma Analysis of Surrogacy Hypothesis (FLASH) analysis with individual patient data (IPD) of 3,837 patients (pts). J Clin Oncol. 2015;33(suppl) (abstract 850). 